\title{
The Probabilistic Deconvolution of the Distribution of Relaxation Times with Finite Gaussian Processes
}

Adeleke Maradesa ${ }^{\mathrm{a}}$, Baptiste Py ${ }^{\mathrm{a}}$, Emanuele Quattrocchi ${ }^{\mathrm{a}}$, Francesco Ciucci $^{\mathrm{a}, \mathrm{b}, *}$

${ }^{a}$ Department of Mechanical and Aerospace Engineering, The Hong Kong University of Science and Technology, Hong Kong, China

${ }^{\mathrm{b}}$ Department of Chemical and Biological Engineering, The Hong Kong University of Science and Technology, Hong Kong, China

*Corresponding author: francesco.ciucci@ust.hk

Phone: +85223587187

Fax: +85223581543 


\begin{abstract}
Electrochemical impedance spectroscopy (EIS) is a tool widely used to study the properties of electrochemical systems. The distribution of relaxation times (DRT) has emerged as one of the main methods for the analysis of EIS spectra. Gaussian processes can be used to regress EIS data, quantify uncertainty, and deconvolve the DRT, but current implementations do not constrain the DRT to be positive and can only use the imaginary part of EIS spectra. Herein, we overcome both issues by using a finite Gaussian process approximation to develop a new framework called the finite Gaussian process distribution of relaxation times (fGP-DRT). The analysis on artificial EIS data shows that the fGP-DRT method consistently recovers exact DRT from noise-corrupted EIS spectra while accurately regressing experimental data. Furthermore, the fGP-DRT framework is used as a machine learning tool to provide probabilistic estimates of the impedance at unmeasured frequencies. The method is further validated against experimental data from fuel cells and batteries. In short, this work develops a novel probabilistic approach for the analysis of EIS data based on Gaussian process, opening a new stream of research for the deconvolution of DRT.
\end{abstract}

Keywords: Electrochemical Impedance Spectroscopy, Distribution of Relaxation Times, Gaussian Processes, Fuel Cells, Lithium-ion Batteries 


\section{Introduction}

Electrochemical impedance spectroscopy (EIS) is commonly used to analyze electrochemical systems [1-10], including sensors [10-12], supercapacitors [13-16], batteries [6,7,15-17] and fuel cells [1,18-23]. Despite the tremendous usefulness of this technique, obtaining meaningful insights from EIS data is difficult. Equivalent circuit models (ECMs) are usually used to analyze EIS data [16,24-27], but ECMs are not unique as several circuits can fit EIS data equally well [1,28-30]. Physical models can provide more insight than ECMs [1,30]. However, physical models are system-specific and difficult to implement. EIS analysis can also be carried out using the distribution of relaxation times (DRT) $[2,8,22,23,26,31-35]$. The DRT framework assumes that EIS spectra result from relaxations, implying that the impedance can be modeled as [1,36-38]

$$
Z_{\mathrm{DRT}}(f)=2 i \pi f L_{0}+R_{\infty}+\int_{-\infty}^{+\infty} \frac{\gamma(\log \tau)}{1+2 i \pi f \tau} d \log \tau
$$

where $L_{0}, R_{\infty}, f$, and $\gamma(\log \tau)$ are the inductance, ohmic resistance, frequency, and DRT, respectively $[1,11]$.

Deconvolving $\gamma(\log \tau)$ from EIS measurements is challenging because an ill-posed inverse problem needs to be solved [1,39-48]. Several approaches, including maximum entropy, genetic algorithms, ridge regression, evolutionary algorithms, elastic net, Ridge and Lasso regression, and Bayesian methods have been used [4,41,43,45-47,49-53]. Among them, probabilistic methods have emerged as the method of choice for DRT deconvolution due to their ability to quantify uncertainty. In that context, our research group recently developed the Gaussian process distribution of relaxation times (GP-DRT) method, which assumes the DRT to be a Gaussian 
process (GP) [1]. The GP-DRT was shown to effectively deconvolve the DRT, fitting both synthetic and real EIS data. One particularly appealing trait of the GP-DRT method is that its hyperparameters can be obtained by maximizing the evidence, ensuring maximal consistency with experiments. The GP-DRT method has, however, two limitations. First, the $\gamma(\log \tau)$ estimated using the GP-DRT is unconstrained and, therefore, can be negative (which is unphysical). Second, the current implementation assumes that only the imaginary part of the impedance spectrum can be used [54]. To overcome these limitations, we developed a new model based on a finite approximation of GPs [55]. Herein, this method will be called the finite Gaussian process distribution of relaxation times (fGP-DRT). The fGP-DRT was set up so that it can recover both the real and imaginary parts of the impedance under the constraint that the DRT is non negative, i.e., $\gamma(\log \tau) \geq 0$. The fGP-DRT method was successfully validated against artificial and real EIS data. Furthermore, like the GP-DRT, the fGP-DRT was used as a machine learning toolbox, allowing the prediction of EIS spectra at unmeasured frequencies. This work extends the interpretation of EIS data through the lens of probabilistic modeling, and will likely inspire more research works that leverage GPs for DRT deconvolution. 


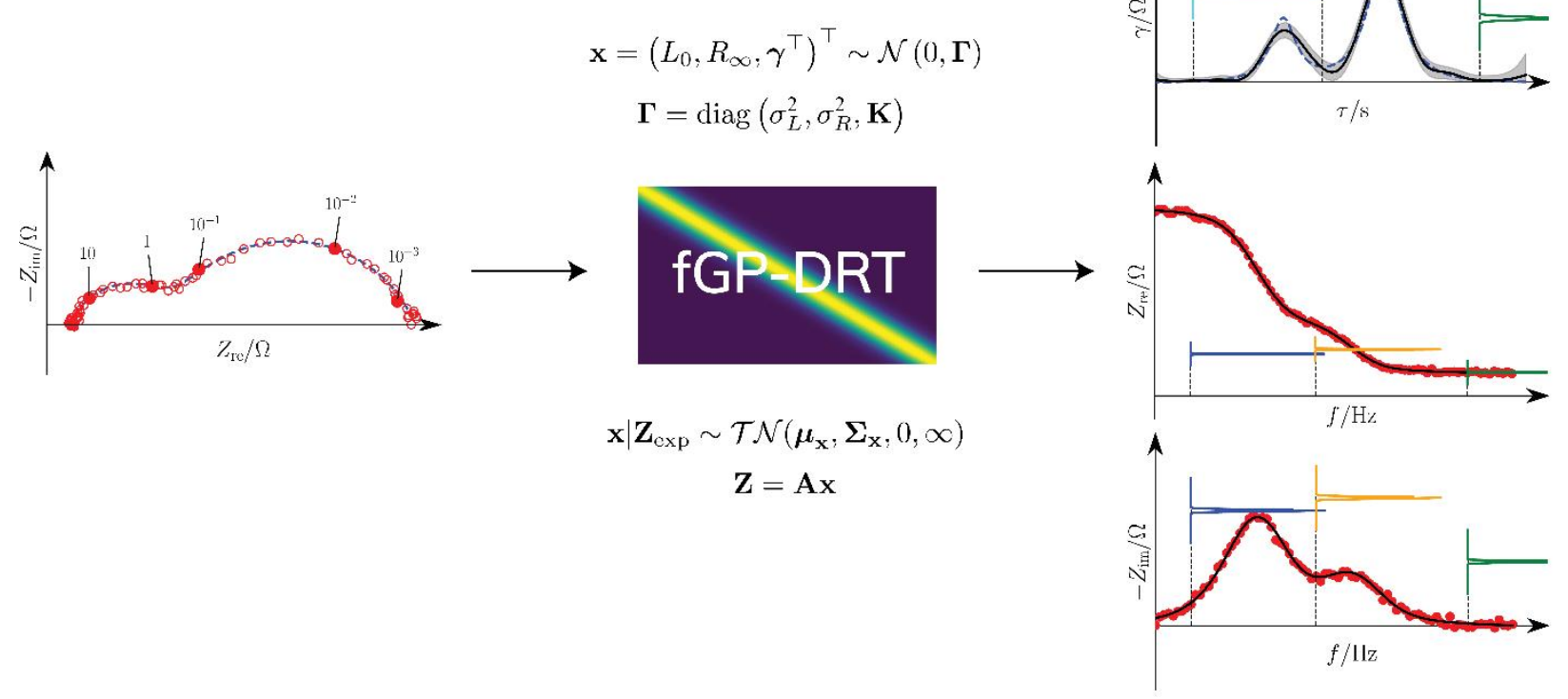

Figure 1: Schematic illustration of the fGP-DRT framework

\section{Theory}

\subsection{Gaussian Process}

A Gaussian process (GP) can be loosely understood as an infinite collection of random variables such that any finite subset of these random variables has a joint Gaussian distribution $[1,56,57]$. We will take the DRT, $\gamma(\xi)$, where $\xi=\log \tau$, to be a GP with mean $m(\xi)$ and kernel $k\left(\xi, \xi^{\prime}\right)$, i.e., $[56,57]$

$$
\gamma(\xi) \sim \mathcal{G P}\left(m(\xi), k\left(\xi, \xi^{\prime}\right)\right)
$$

A finite-dimensional approximation, $\gamma_{N}(\xi)$, of $\gamma(\xi)$ can be defined as follows:

$$
\gamma_{N}(\xi)=\sum_{n=1}^{N} \gamma\left(\xi_{n}\right) \phi_{n}(\xi)
$$


where $\xi_{1} \leq \xi_{2} \leq \ldots \leq \xi_{N}$ are equispaced collocation points with $\xi_{n+1}-\xi_{n}=\Delta$ for $n=$ $1,2, \ldots, N-1$, and $\phi_{n}(\xi)$ is the following triangular function

$$
\phi_{n}(\xi)=\left\{\begin{array}{cc}
1-\frac{\left|\xi-\xi_{n}\right|}{\Delta} & \text { if }\left|\xi-\xi_{n}\right|<\Delta \\
0 & \text { otherwise }
\end{array}\right.
$$

By defining $\gamma=\left(\gamma\left(\xi_{1}\right), \gamma\left(\xi_{2}\right), \ldots, \gamma\left(\xi_{N}\right)\right)^{\top}$ and $\xi=\left(\xi_{1}, \xi_{2}, \ldots, \xi_{N}\right)^{\top}$, and setting $m\left(\xi_{n}\right)=0$ for $n=1,2, \ldots, N$ (or in short-hand notation $m(\xi)=\mathbf{0}$ ), it follows that

$$
\boldsymbol{\gamma} \sim \mathcal{N}(\mathbf{0}, \boldsymbol{K})
$$

where $(\boldsymbol{K})_{m n}=k\left(\xi_{m}, \xi_{n}\right)$ for $m, n=1,2, \ldots, N$ (or in short-hand notation $\boldsymbol{K}=k(\xi, \xi)$ ). The DRT model (1) can be written as

$$
\begin{gathered}
Z_{\mathrm{re}}(f)=R_{\infty}+\int_{-\infty}^{+\infty} \frac{1}{1+\left(2 \pi f e^{\xi}\right)^{2}} \gamma(\xi) d \xi \\
Z_{\mathrm{im}}(f)=2 \pi L_{0} f-\int_{-\infty}^{+\infty} \frac{2 \pi f e^{\xi}}{1+\left(2 \pi f e^{\xi}\right)^{2}} \gamma(\xi) d \xi
\end{gathered}
$$

By replacing $\gamma(\xi)$ with the $\gamma_{N}(\xi)$ given in (3), we can rewrite (6) as

$$
\begin{gathered}
Z_{\mathrm{re}}(f)=R_{\infty}+\mathbf{a}_{\mathrm{re}}(f)^{\top} \boldsymbol{\gamma} \\
Z_{\mathrm{im}}(f)=2 \pi L_{0} f+\mathbf{a}_{\mathrm{im}}(f)^{\top} \boldsymbol{\gamma}
\end{gathered}
$$

where

$$
\begin{gathered}
\left(\mathbf{a}_{\mathrm{re}}(f)\right)_{n}=\int_{-\infty}^{+\infty} \frac{1}{1+\left(2 \pi f e^{\xi}\right)^{2}} \phi_{n}(\xi) d \xi \\
\left(\mathbf{a}_{\mathrm{im}}(f)\right)_{n}=-\int_{-\infty}^{+\infty} \frac{2 \pi f e^{\xi}}{1+\left(2 \pi f e^{\xi}\right)^{2}} \phi_{n}(\xi) d \xi
\end{gathered}
$$


From (7) and (8), the real and imaginary parts of the impedance can be computed at frequencies $\boldsymbol{f}=\left(f_{1}, f_{2}, \ldots, f_{M}\right)^{\top}$ as

$$
\begin{gathered}
\boldsymbol{Z}_{\mathrm{re}}(\boldsymbol{f})=\mathrm{R}_{\infty} \mathbf{1}+\mathbf{A}_{\mathrm{re}} \boldsymbol{\gamma} \\
\boldsymbol{Z}_{\mathrm{im}}(\boldsymbol{f})=2 \pi L_{0} \boldsymbol{f}+\mathbf{A}_{\mathrm{im}} \boldsymbol{\gamma}
\end{gathered}
$$

where $\mathbf{A}_{\mathrm{re}}$ and $\mathbf{A}_{\mathrm{im}}$ are built by stacking the vectors $\mathbf{a}_{\mathrm{re}}(f)$ and $\mathbf{a}_{\mathrm{im}}(f)$, respectively, i.e., $\left(\mathbf{A}_{\mathrm{re}}\right)_{m, \cdot}=\mathbf{a}_{\mathrm{re}}\left(f_{m}\right)^{\top}$ and $\left(\mathbf{A}_{\mathrm{im}}\right)_{m, \cdot}=\mathbf{a}_{\mathrm{im}}\left(f_{m}\right)^{\top}$ for $m=1,2, \ldots, M$.

After defining $\boldsymbol{Z}=\left(\begin{array}{c}Z_{\mathrm{re}} \\ Z_{\mathrm{im}}\end{array}\right)$ and $\boldsymbol{A}=\left(\begin{array}{ccc}\mathbf{0} & \mathbf{1} & \mathbf{A}_{\mathrm{re}} \\ 2 \pi \boldsymbol{f} & \mathbf{0} & \mathbf{A}_{\mathrm{im}}\end{array}\right)$, we assume that the experimental impedance is given by

$$
Z=A x+\varepsilon
$$

where $\boldsymbol{x}=\left(L_{0}, R_{\infty}, \gamma(\xi)^{\top}\right)^{\top}, \boldsymbol{\varepsilon} \sim \mathcal{N}\left(\mathbf{0}, \sigma_{n}^{2} \mathbf{I}\right), \sigma_{n}$ is the standard deviation of the random error, and $\mathbf{I}$ is the $2 M \times 2 M$ identity matrix. We now suppose that

$$
\boldsymbol{x} \sim \mathcal{N}(0, \Gamma)
$$

with

$$
\boldsymbol{\Gamma}=\left(\begin{array}{ccc}
\sigma_{L}^{2} & 0 & 0 \\
0 & \sigma_{R}^{2} & 0 \\
0 & 0 & \boldsymbol{K}
\end{array}\right)
$$

where $\sigma_{L}$ and $\sigma_{R}$ are the standard deviations of $L_{0}$ and $R_{\infty}$, respectively $\left(L_{0} \sim \mathcal{N}\left(0, \sigma_{L}^{2}\right)\right.$ and $R_{\infty} \sim$ $\left.\mathcal{N}\left(0, \sigma_{R}^{2}\right)\right)$. It follows from (10) and (11) that the joint distribution of $\boldsymbol{x}$ and $\boldsymbol{Z}$ is the following multivariate Gaussian: 


$$
\left(\begin{array}{l}
\boldsymbol{x} \\
\boldsymbol{Z}
\end{array}\right) \sim \mathcal{N}\left(\mathbf{0},\left(\begin{array}{cc}
\boldsymbol{\Gamma} & \boldsymbol{\Gamma} \boldsymbol{A}^{\top} \\
\boldsymbol{A} \boldsymbol{\Gamma} & \boldsymbol{A} \boldsymbol{\Gamma} \boldsymbol{A}^{\top}+\sigma_{\mathrm{n}}^{2} \mathbf{I}
\end{array}\right)\right)
$$

Using (13), we can compute the distribution of $\boldsymbol{x}$ conditioned to the experimental data $\boldsymbol{Z}_{\exp }[1,56]$ :

$$
\boldsymbol{x} \mid \boldsymbol{Z}=\boldsymbol{Z}_{\text {exp }} \sim \mathcal{N}\left(\boldsymbol{\mu}_{\mathrm{x}}, \boldsymbol{\Sigma}_{\mathrm{x}}\right)
$$

where

$$
\begin{gathered}
\boldsymbol{\mu}_{\mathrm{x}}=\boldsymbol{\Gamma} \boldsymbol{A}^{\top}\left(\boldsymbol{A} \boldsymbol{\Gamma} \boldsymbol{A}^{\top}+\sigma_{n}^{2} \mathbf{I}\right)^{-1} \boldsymbol{Z}_{\mathrm{exp}} \\
\boldsymbol{\Sigma}_{\mathrm{x}}=\boldsymbol{\Gamma}-\boldsymbol{\Gamma} \boldsymbol{A}^{\top}\left(\boldsymbol{A} \boldsymbol{\Gamma} \boldsymbol{A}^{\top}+\sigma_{n}^{2} \mathbf{I}\right)^{-1} \boldsymbol{A} \boldsymbol{\Gamma}
\end{gathered}
$$

\subsection{Positivity Constraint}

As outlined in the introduction, the current GP-DRT model does not impose any constraint on $\boldsymbol{x}$ [1].

However, starting from (14), it is straightforward to impose a constraint on $\boldsymbol{x} \mid \boldsymbol{Z}$ by taking that $\boldsymbol{x} \mid \boldsymbol{Z}$ is a truncated multinormal distribution bound by zero from below, i.e.,

$$
\boldsymbol{x} \mid \boldsymbol{Z}=\boldsymbol{Z}_{\mathrm{exp}} \sim \mathcal{T} \mathcal{N}\left(\boldsymbol{\mu}_{\mathrm{x}}, \boldsymbol{\Sigma}_{\mathrm{x}}, 0, \infty\right)
$$

To sample $\boldsymbol{x}$ from the truncated multinormal distribution (14), Hamiltonian Monte Carlo sampling can be used [58]. We generated 10,000 samples and discarded the first 1,000 as burn-in. The impedance can then be obtained from the sampled $\boldsymbol{x}$ by matrix multiplication, i.e., $\boldsymbol{Z}=\boldsymbol{A x}$. In the figures in the remainder of the article, the means of DRT and impedance are shown as a black line, while the $3 \times \sigma$ credible intervals are shown as gray regions.

\subsection{Kernel and Hyperparameter Selection}

To compute the matrix $\boldsymbol{K}$ reported in (5), we used the squared-exponential kernel, $k\left(\xi, \xi^{\prime}\right)$, defined as $[1,59]$ 


$$
k\left(\xi, \xi^{\prime}\right)=\sigma_{f}^{2} \exp \left(-\frac{1}{2 \ell^{2}}\left(\xi-\xi^{\prime}\right)^{2}\right)
$$

where $\sigma_{f}$ and $\ell$ are two scalars. Therefore, the vector of hyperparameters is $\boldsymbol{\theta}=\left(\sigma_{n}, \sigma_{L}, \sigma_{R}, \sigma_{f}, \ell\right)^{\top}$. Unless otherwise specified, the values of $\boldsymbol{\theta}$ were obtained by maximizing the experimental evidence [60], i.e., $p\left(\boldsymbol{Z}=\boldsymbol{Z}_{\text {exp }} \mid \boldsymbol{\theta}\right)$, given by

$$
\begin{gathered}
\log \left(p\left(\boldsymbol{Z}=\boldsymbol{Z}_{\text {exp }} \mid \boldsymbol{\theta}\right)\right) \\
=-\frac{1}{2} \boldsymbol{Z}_{\exp }^{\top}\left(\boldsymbol{A} \boldsymbol{\Gamma} \boldsymbol{A}^{\top}+\sigma_{n}^{2} \mathbf{I}\right)^{-1} \boldsymbol{Z}_{\text {exp }}-\frac{1}{2} \log \left(\left|\boldsymbol{A} \boldsymbol{\Gamma} \boldsymbol{A}^{\top}+\sigma_{n}^{2} \mathbf{I}\right|\right) \\
-\frac{(N+2)}{2} \log (2 \pi)
\end{gathered}
$$

In the actual implementation, $L(\boldsymbol{\theta})=-\log \left(p\left(\boldsymbol{Z}=\boldsymbol{Z}_{\text {exp }} \mid \boldsymbol{\theta}\right)\right)-\frac{(N+2)}{2} \log (2 \pi)$ was minimized.

\section{Results}

We first validated the consistency of the fGP-DRT model against artificial experiments generated by corrupting exact EIS spectra characterized by known DRTs. Then, the model was tested against real EIS data of fuel cells and batteries.

\subsection{Artificial experiments}

All artificial EIS data were generated, unless otherwise specified, using log-spaced frequencies in the $10^{-4}-10^{4} \mathrm{~Hz}$ range with 10 points per decade. We first considered a single ZARC model, for which we analyzed the influence of the experimental error, the number of collocation points ( $N$ as defined in equation (3)), and truncated spectra. After that, we studied models with overlapping (2×ZARC) and discontinuous timescales (piecewise constant and fractal elements). 


\subsubsection{Single ZARC Model}

A single ZARC circuit is an ohmic resistor $\left(R_{\infty}\right)$ in series with a parallel circuit consisting of a resistor $\left(R_{\mathrm{ct}}\right)$ and a constant phase element $(\mathrm{CPE})$, with characteristic time $\tau_{0}$ and parameter $\phi$. The impedance, $Z_{\text {exact }}(f)$, and its corresponding DRT are given by $[1,4]$

$$
\begin{gathered}
Z_{\text {exact }}(f)=R_{\infty}+\frac{R_{\mathrm{ct}}}{1+\left(i 2 \pi f \tau_{0}\right)^{\phi}} \\
\gamma(\log \tau)=\frac{R_{\mathrm{ct}}}{2 \pi} \frac{\sin ((1-\phi) \pi)}{\cosh \left(\phi \log \left(\tau / \tau_{0}\right)\right)-\cos (\pi(1-\phi))}
\end{gathered}
$$

and the parameters used in the model are reported in Table 1. For the synthetic experiments, we generated data using (12) where the error standard deviation is given by $\sigma_{n}^{\exp }=0.5 \Omega^{1}$. The Nyquist plots of the exact and noise-corrupted impedance spectra are shown in Figure 2(a). The DRT was recovered with the fGP-DRT without and with non-negativity constraint (Figure 2(b) and (c), respectively). As expected, the non-negativity constraint has the effect of narrowing the credible bands. The real and imaginary parts of the impedance are also shown for the fGP-DRT model under non-negativity constraint (Figure 2(d)), highlighting the excellent recovery of the EIS data.

Table 1- Parameters used for the exact impedance of the single ZARC model.

\begin{tabular}{|c|c|}
\hline Parameter & Value \\
\hline$R_{\infty}$ & $10 \Omega$ \\
\hline$R_{\text {ct }}$ & $50 \Omega$ \\
\hline$\tau_{0}$ & $1 \mathrm{~s}$ \\
\hline$\phi$ & 0.8 \\
\hline$L_{0}$ & $5.0 \times 10^{-4} \mathrm{H}$ \\
\hline
\end{tabular}

${ }^{1}$ Note that $\sigma_{n}^{\exp }$ is the standard deviation of the noise in the artificial experiments while $\sigma_{n}$ is a hyperparameter of the fGP-DRT method. 
We also considered a single ZARC plus an inductor (values of the parameters in Table 1), whose Nyquist and Bode plots for both unconstrained and constrained DRT are reported in Figure S1 of the SI. We observe that the unconstrained fGP-DRT oscillates about the exact DRT with widening error bands for $\tau>10^{3} \mathrm{~s}$ (Figure S1(b)). Instead, the constrained fGP-DRT model exhibits a better recovery of $\gamma(\log \tau)$. The real and imaginary components of the impedance obtained using the constrained fGP-DRT match well with the exact impedance spectrum.

(a)

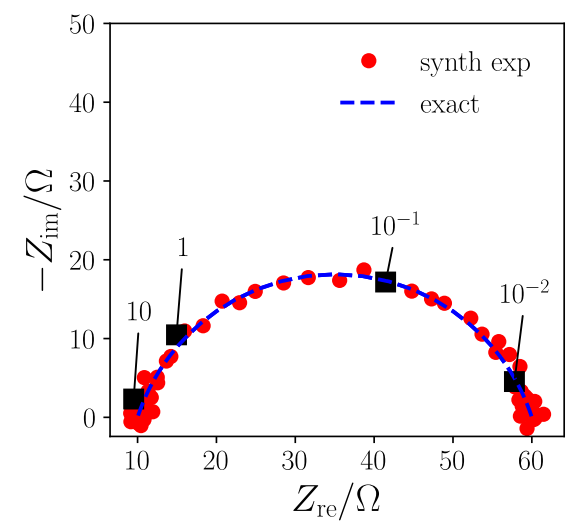

(c)

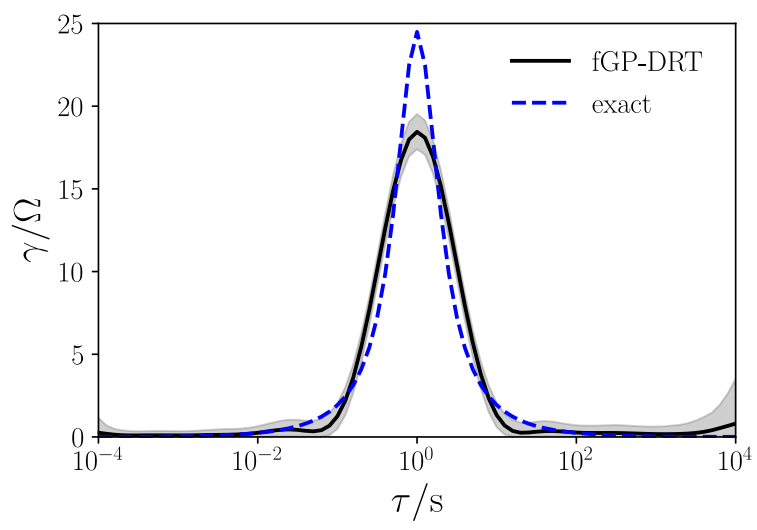

(b)

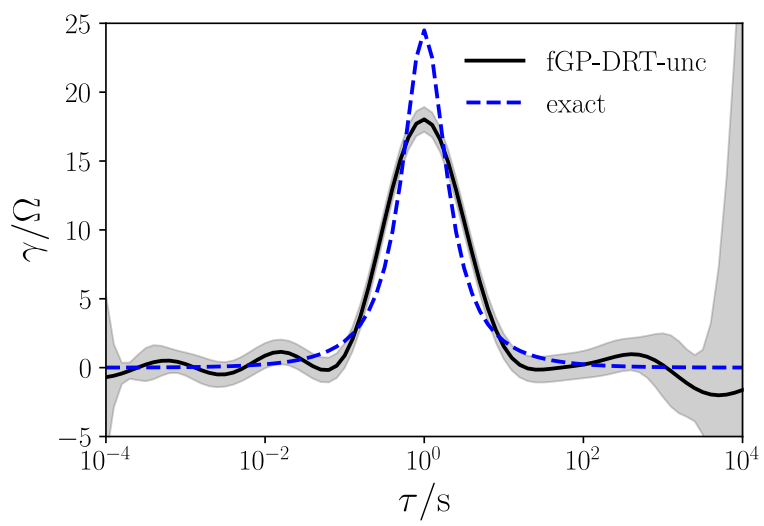

(d)

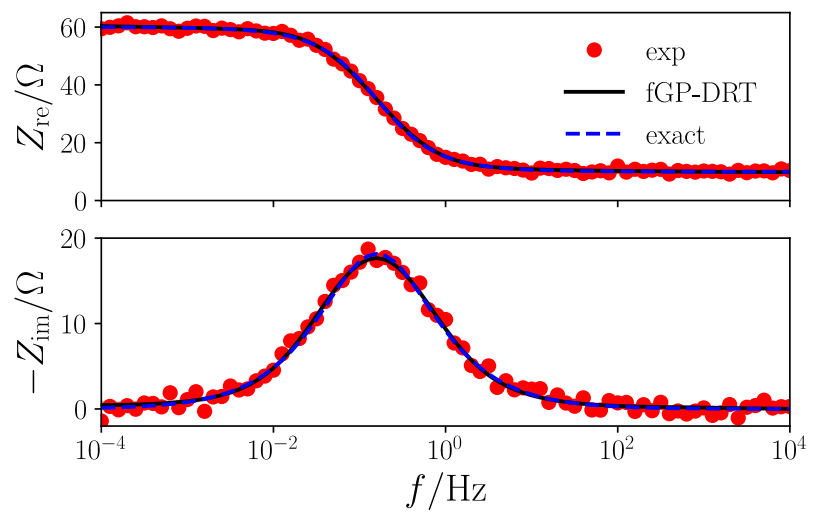

Figure 2: (a) Nyquist plot of a single ZARC with experimental noise standard deviation $\sigma_{n}^{\text {exp }}=$ $0.5 \Omega$. Recovered (b) unconstrained, and (c) constrained DRT. (d) Regressed real and imaginary parts of the impedance under non-negativity constraint. 


\subsubsection{Hyperparameter Selection}

The hyperparameters (which are stored in the vector $\boldsymbol{\theta}$ ) have a remarkable impact on the recovery of DRT and impedance. To illustrate their influence, we applied the fGP-DRT method for specific values. We first set $\sigma_{f}=\sigma_{L}=1.0, \sigma_{R}=2.0, \sigma_{n}=0.5$, and varied $\ell\left(10^{-2}, 0.5,1.0\right.$, and 2.0). The estimated DRT (with its credible bands included) and recovered impedance are given in the left and right panels of Figure 3, respectively. For $\ell=10^{-2}$ (Figure 3 (a)), the estimated DRT displays a large variance, as shown by the shaded region around the mean. The credible bands reduce, and the peak values of the recovered impedance become closer to the exact values as $\ell$ increases to 1.0, as shown in Figure 3(d) and (f). To understand why this happens, $\boldsymbol{K}$ was plotted as a function

of $\ell$ as shown in Figure S2. The matrix $K$ is nearly diagonal for $\ell=10^{-2}$, indicating that a value of $\gamma(\log \tau)$ at a particular $\log \tau$ is only influenced by adjacent $\log$ timescales. In contrast, we note in Figure $\mathrm{S} 2$ a strong dependency across a broader ranges of timescales as $\ell$ increases. The results reported in Figure S3, which shows the recovered real and imaginary components of the impedance, the estimated DRT and the values of the entries in the matrix $K$ for small $\left(\ell=10^{-5}\right)$ and moderately high ( $\ell=2.5$ and 5.0) length scales, also support these conclusions and highlight the strong influence of the kernel and, in particular, the correlation log-timescale $\ell$ on the recovered DRT. In the remainder of the article, the hyperparameters are obtained by optimizing the experimental evidence, that is the probability of the experimental data given the model. 
(a)

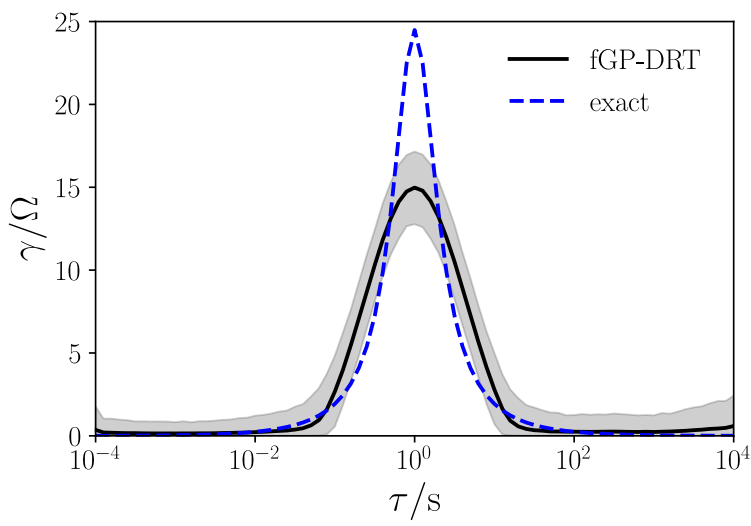

(c)

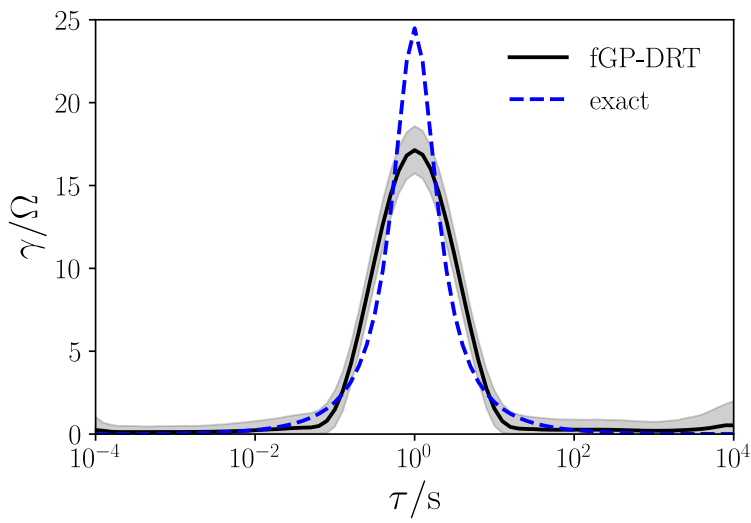

(e)

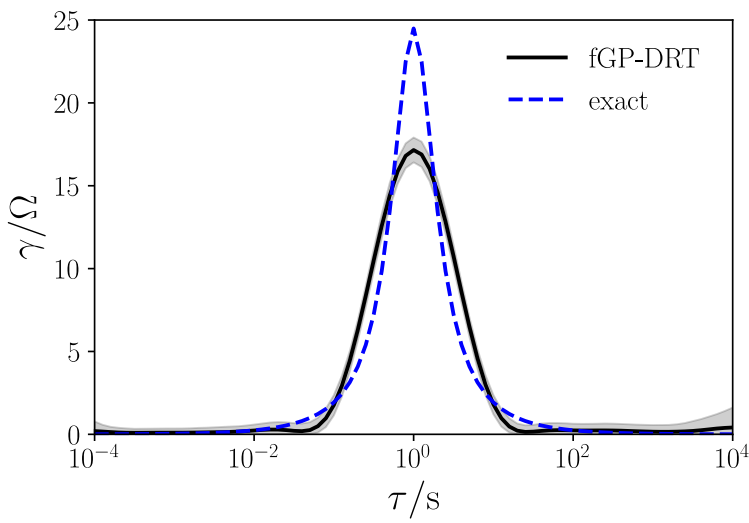

(b)
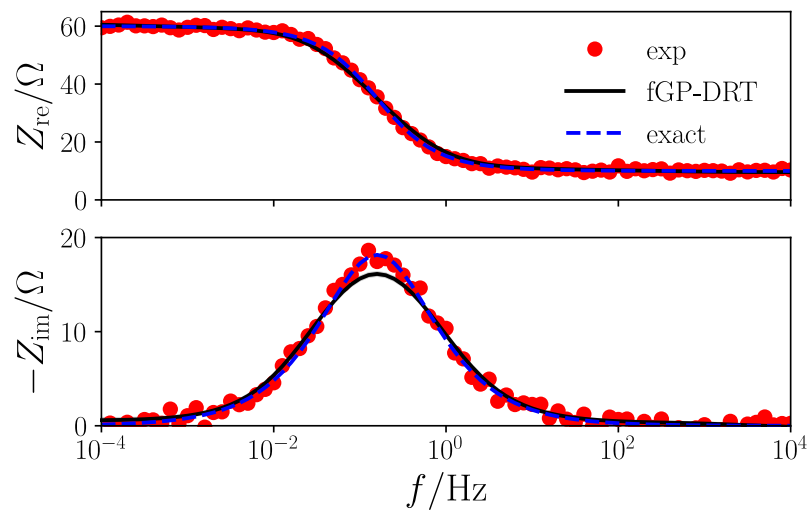

(d)
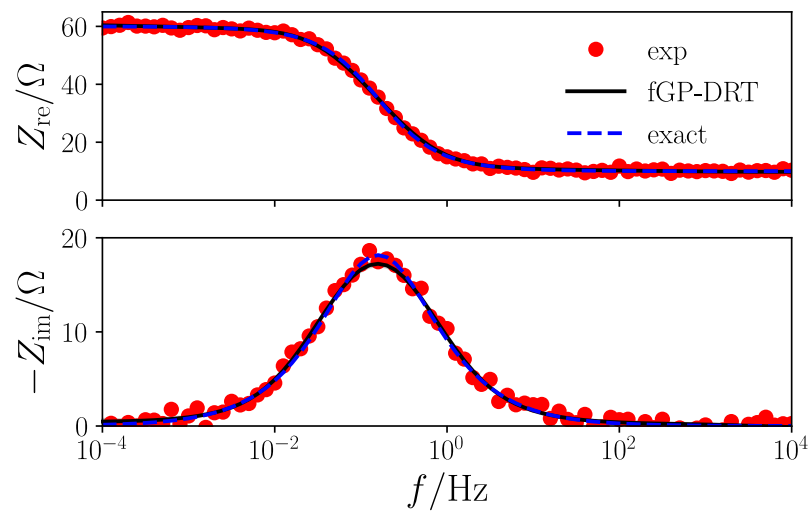

(f)

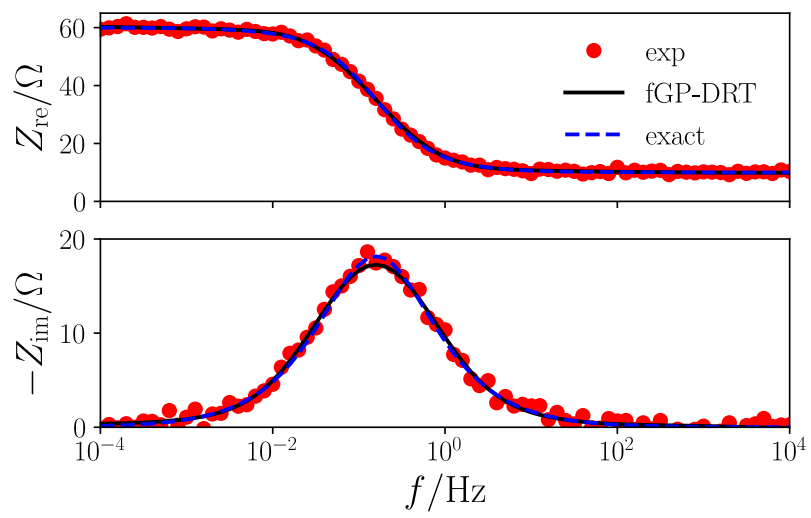

Figure 3: The recovered DRT under the non-negatively constrained fGP-DRT model for $\ell$ equal to (a) $10^{-2}$, (b) 0.5 , and (c) 1.0 with $\sigma_{f}=\sigma_{L}=1.0$ and $\sigma_{R}=2.0$. The corresponding real and imaginary components of the impedance are shown in panels in (b), (d), and (f), respectively. 


\subsubsection{Influence of the Experimental Errors}

We now illustrate the influence of the experimental noise on the predictions produced by the fGPDRT model. The hyperparameters were obtained by maximizing the experimental evidence at specified noise levels, $\sigma_{n}^{\text {exp }}$. Panels (a), (d), and (g) of Figure 4 show the Nyquist plots corresponding to $\sigma_{n}^{\exp }=1.0,2.0$, and $3.0 \Omega$, respectively. As $\sigma_{n}^{\text {exp }}$ increases, the recovered DRT has larger credible bands around the mean, indicating greater uncertainty, see panels (b), (e), and (h) of Figure 4. There is, in particular, a significant widening of the credible band at high timescales $\left(\tau>10^{4} \mathrm{~s}\right)$

Using equation (10), 1,000 synthetic experiments for each $\sigma_{n}^{\exp }=0.2, \ldots, 2.0 \Omega$, were generated; the optimal $\boldsymbol{\theta}$ was obtained for each synthetic experiment. The obtained $\sigma_{n}$ and $\sigma_{R}$ are shown in Figure 5 as a boxplot, where the notch indicates the median, the box the $25-75 \%$ interquartile range, the whiskers 1.5 times that interquartile, and the circles the outliers. The upper and lower quantiles increase with increasing $\sigma_{n}^{\exp }$, meaning an increase uncertainty consistent with expectations. Unsurprisingly, the mean of $\sigma_{n}$ is very close to $\sigma_{n}^{\exp }$ and the mean of $\sigma_{R}$ is independent of $\sigma_{n}^{\text {exp }}$. 
(a)

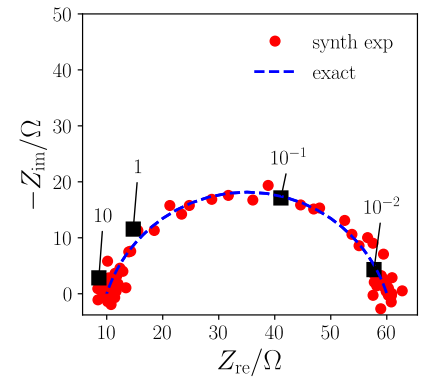

(d)

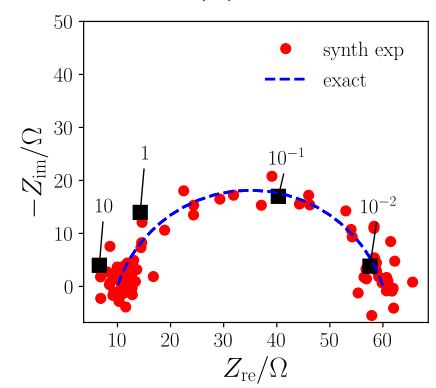

(g)

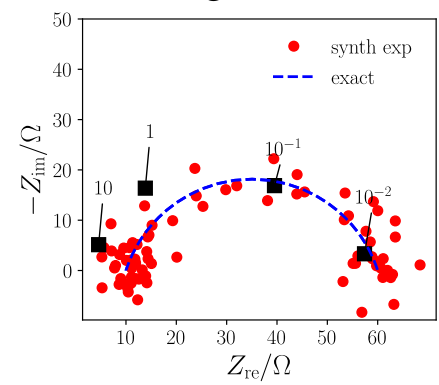

(b)

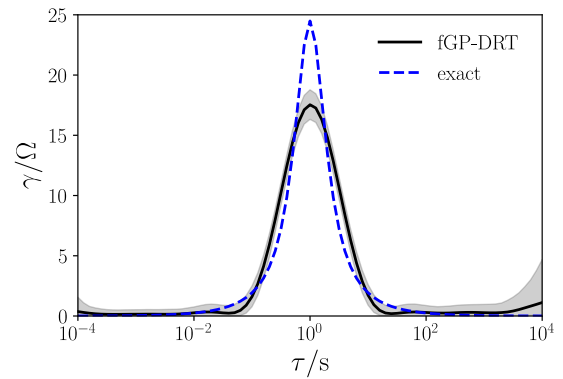

(e)

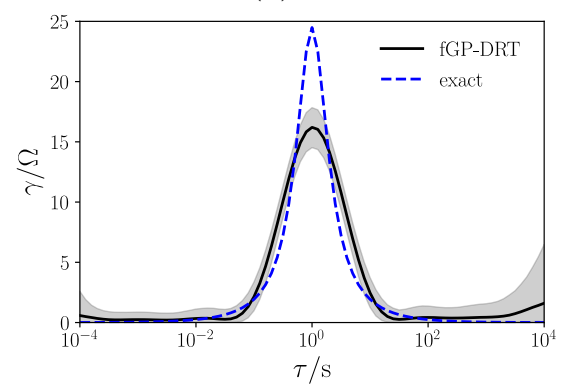

(h)

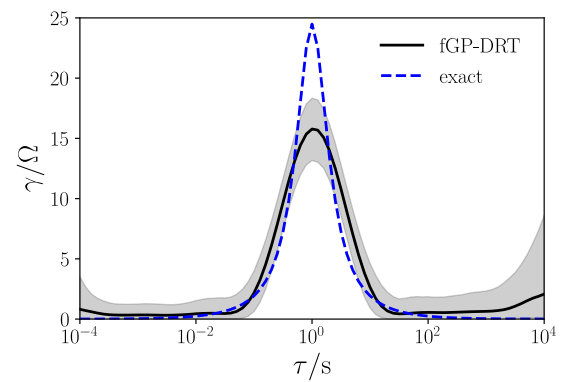

(c)
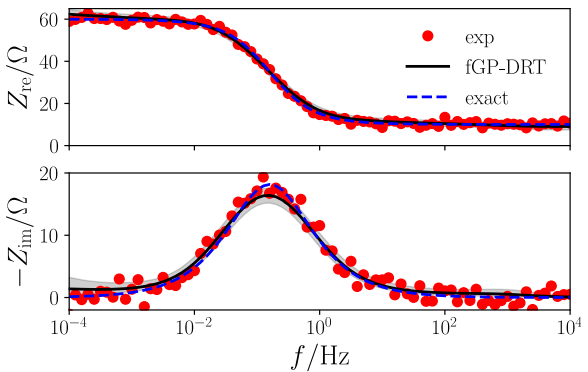

(f)

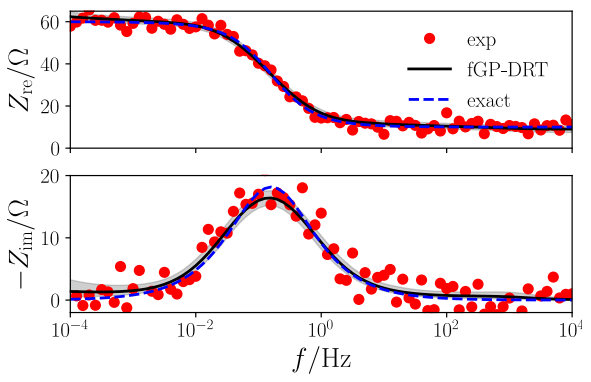

(i)

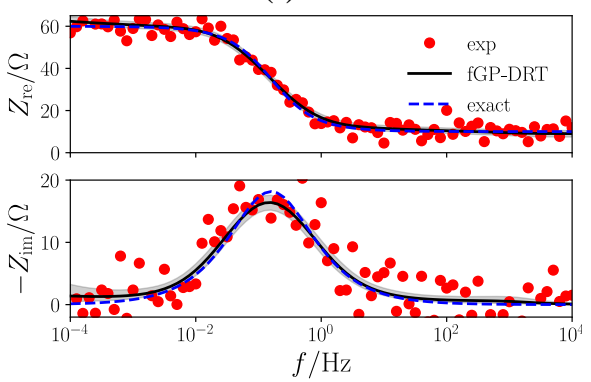

Figure 4: Nyquist plot for a single ZARC with $\sigma_{n}^{\text {exp }}=1.0,2.0$, and $3.0 \Omega$, (a), (d), and (g), respectively. Corresponding recovered DRT under the non-negativiy constraint (b), (e), and (h), and real and imaginary parts of the impedance (c), (f), and (i). 
(a)

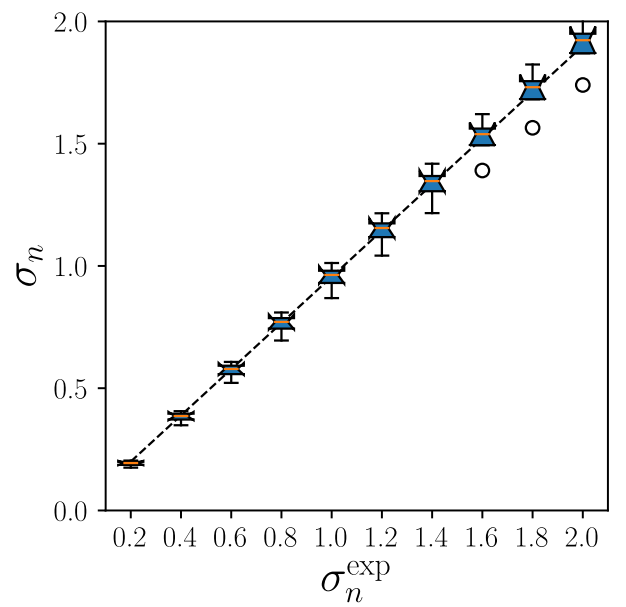

(b)

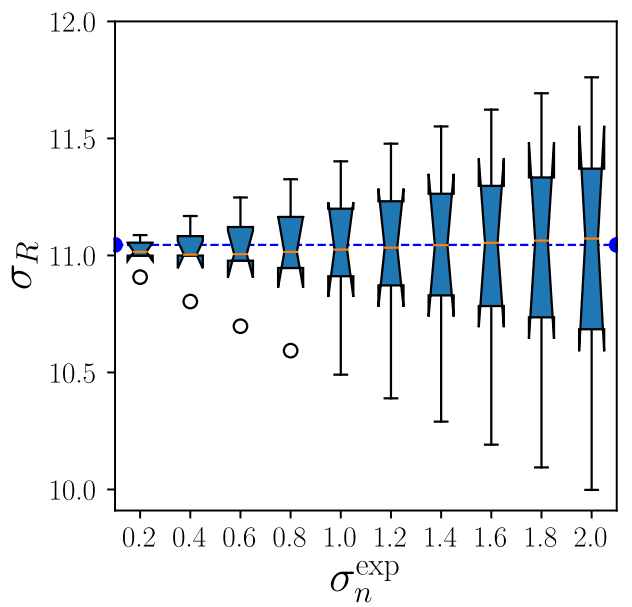

Figure 5: Boxplot of the hyperparameter (a) $\sigma_{n}$ and (b) $\sigma_{R}$ obtained by minimizing $L(\boldsymbol{\theta})$ for 10,000 artificial experiments $\left(1,000\right.$ for each $\left.\sigma_{n}^{\exp }\right)$.

\subsubsection{Data Truncation}

We tested how well the constrained fGP-DRT model deconvolves the DRT when the EIS data is truncated relative to those used in Sections 3.1.1.1 and 3.1.1.2. In particular, the artificial impedance shown in Figure 2 was restricted to frequencies above $10^{-3}, 10^{-2}$, and $10^{-1} \mathrm{~Hz}$ with identical number of points per decade, as shown in Figure 6(a), (b), and (c), respectively. The fGPDRT model successfully recovers both the DRT and the impedance. As expected, the credible bands of the estimated DRT increase for timescales smaller than the truncation threshold, see Figure 6(d), (e), and (f).

For further validation, we considered the ECM described in [61] with the data being truncated below $10^{-3} \mathrm{~Hz}$. The specific parameters of this ECM are reported in Table S2. Our model successfully recovers the real and imaginary components of the impedance. Additionally, the DRT is estimated in a satisfactory manner together with its error bands (Figure S5). 
(a)

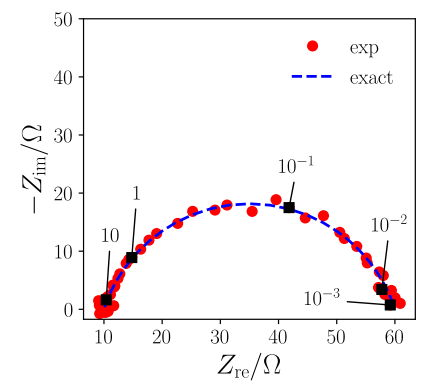

(d)

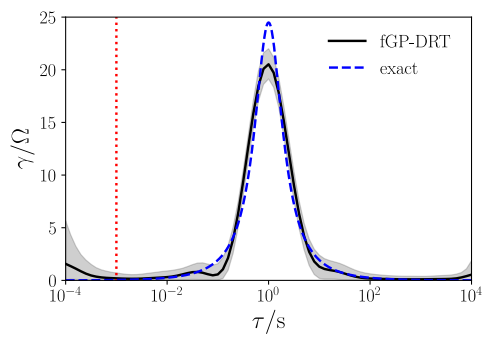

(g)
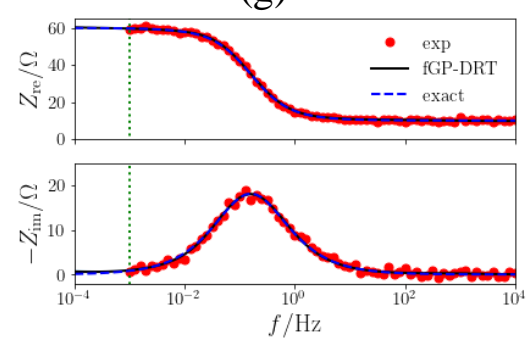

(b)

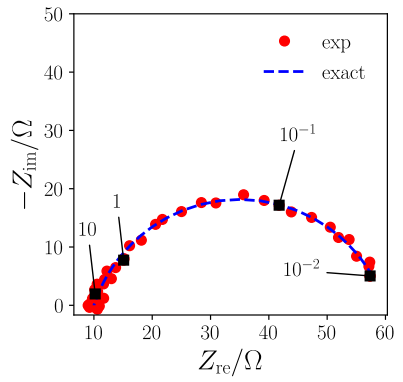

(e)

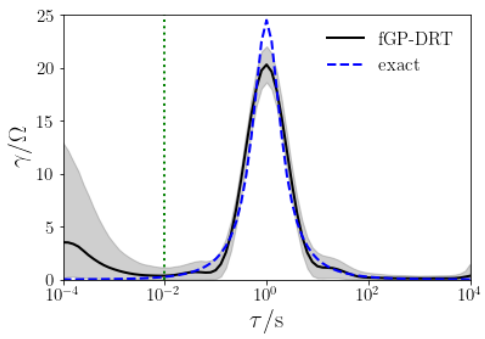

(h)
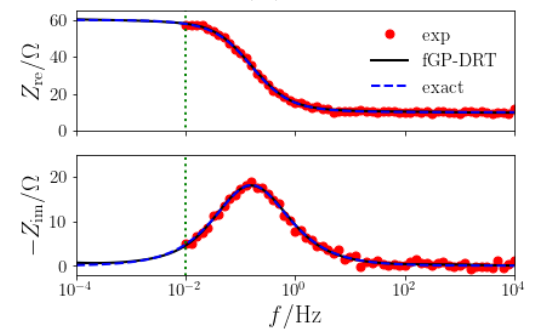

(c)

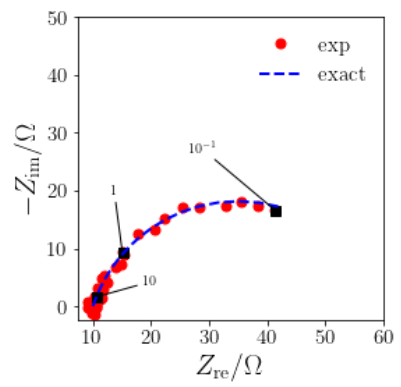

(f)

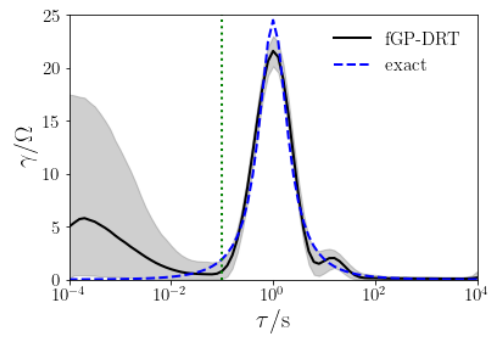

(i)
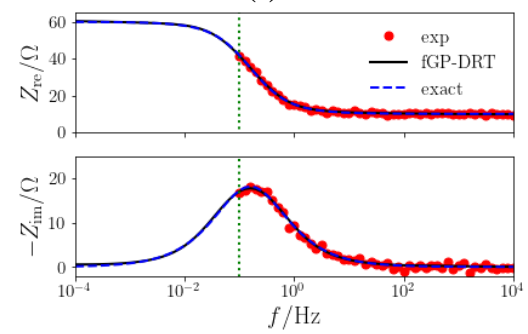

Figure 6: Nyquist plot of the artificial EIS data truncated below (a) $10^{-3}$, (b) $10^{-2}$, and (c) $10^{-1}$ $\mathrm{Hz}\left(\sigma_{n}^{\exp }=0.5 \Omega\right)$ relative to the data presented in Figure 2 with the corresponding recovered DRT in (d), (e), and (f), and real and imaginary parts of the impedance in (g), (h), and (i). 


\subsubsection{Error of Estimation and Discrepancies}

We also explored the influence of the number of collocation points, $N$ in (3), on the mean of the recovered DRT. To that end, we computed the normalized squared residuals against the mean, $r^{2}$, $[49,62]$ defined as

$$
r^{2}=\frac{\left\|\gamma_{\text {exact }}-\hat{\mu}_{\gamma}\right\|^{2}}{\left\|\gamma_{\text {exact }}\right\|^{2}}
$$

where $\hat{\mu}_{\gamma}$ is the value of the mean DRT obtained by sampling (16) using a synthetic EIS spectrum.

We generated 100 artificial experiments, 10 each for $N=20,40,60, \ldots, 200$, and computed the normalized squared residuals $r^{2}$ for each. Figure 7(a) shows the boxplot of $r^{2}$ as a function of $N$. As $N$ increases, the descrepancies significantly reduce, evidenced by the smaller box for higher values of $N$, leading to an improved DRT recovery (Figure 7(b)). Specifically, the curve of the recovered DRT becomes smoother as $N$ increases (Figure S6). A representative recovered DRT for $N=200\left(r^{2}=8.25 \times 10^{-5}\right)$ is shown in Figure 7(b).

(a)

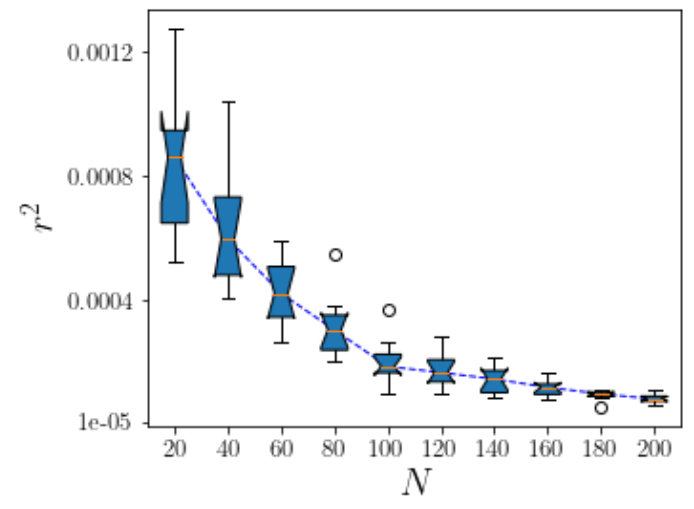

(b)

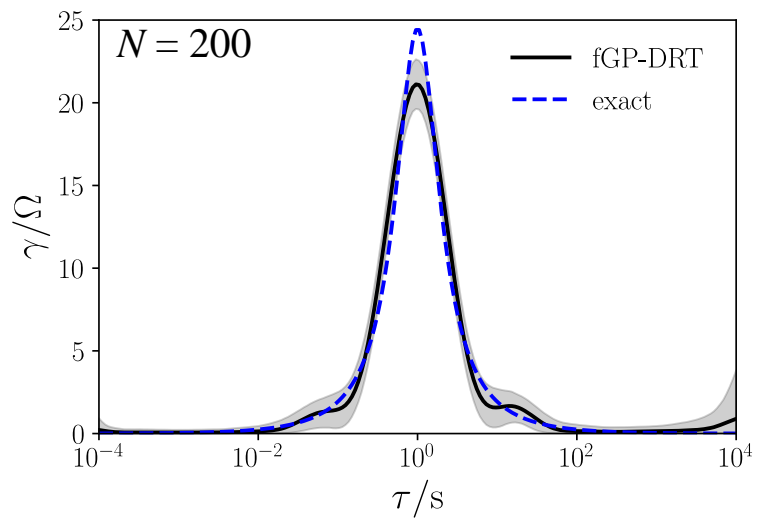

Figure 7: (a) Residual $r^{2}$ against the mean as a function of $N$; (b) recovered DRT using the fGPDRT model for $N=200$. 


\subsubsection{2×ZARC Model}

In many electrochemical systems, numerous physical processes take place concurrently. Therefore, we investigated the capability of the fGP-DRT model to capture overlapping features by studying a $2 \times Z$ ZARC model. The DRT and impedance response are given by $[1,5,49]$

$$
\begin{aligned}
& Z_{\text {exact }}(f)=R_{\infty}+\frac{R_{\mathrm{ct}, 1}}{1+\left(i 2 \pi f \tau_{1}\right)^{\phi_{1}}}+\frac{R_{\mathrm{ct}, 2}}{1+\left(i 2 \pi f \tau_{2}\right)^{\phi_{2}}} \\
& \gamma(\log \tau)=\frac{R_{\mathrm{ct}, 1}}{2 \pi} \frac{\sin \left(\left(1-\phi_{1}\right) \pi\right)}{\cosh \left(\phi_{1} \log \left(\tau / \tau_{1}\right)\right)-\cos \left(\pi\left(1-\phi_{1}\right)\right)} \\
& +\frac{R_{\mathrm{ct}, 2}}{2 \pi} \frac{\sin \left(\left(1-\phi_{2}\right) \pi\right)}{\cosh \left(\phi_{2} \log \left(\tau / \tau_{2}\right)\right)-\cos \left(\pi\left(1-\phi_{2}\right)\right)}
\end{aligned}
$$

where the specific parameters used are reported in Table S1. We set the value $\tau_{1}=0.1 \mathrm{~s}$ and investigated two values for $\tau_{2}$, namely $\tau_{2}=1$ and $10 \mathrm{~s}$. Figure 8 shows the DRT, real, and imaginary parts of the impedance. For separated timescales with $\tau_{1}=0.1 \mathrm{~s}$ and $\tau_{2}=10 \mathrm{~s}$, the Nyquist plot consists in two semicircles (Figure 8(a)). The DRT recovered using the fGP-DRT method correctly shows two distinct peaks (Figure 8(c)). Similarly, for $\tau_{1}=0.1 \mathrm{~s}$ and $\tau_{2}=1.0 \mathrm{~s}$, the Nyquist plot of the impedance resembles a single semicircle with close peaks in the DRT as shown in Figure 8(b) and (d), respectively. The impedance is well regressed as shown in Figure 8(a), (b), (e) and (f). As for the DRT, the location of the peaks is accurately identified, but the credible bands widen as the timescales grow closer (Figure 8(c) and (d)) 
(a)

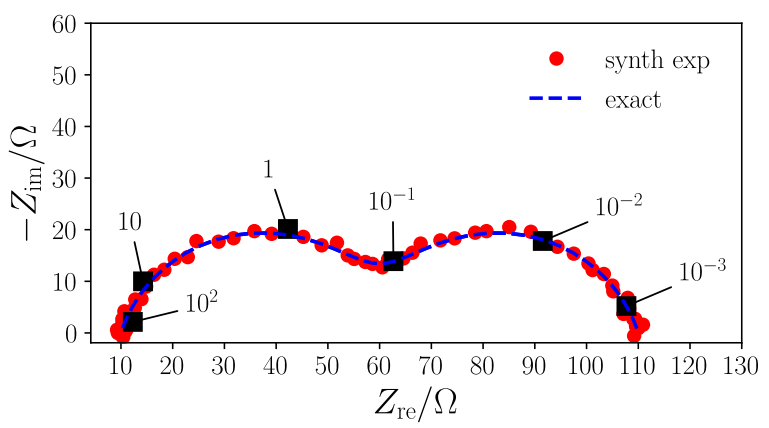

(c)

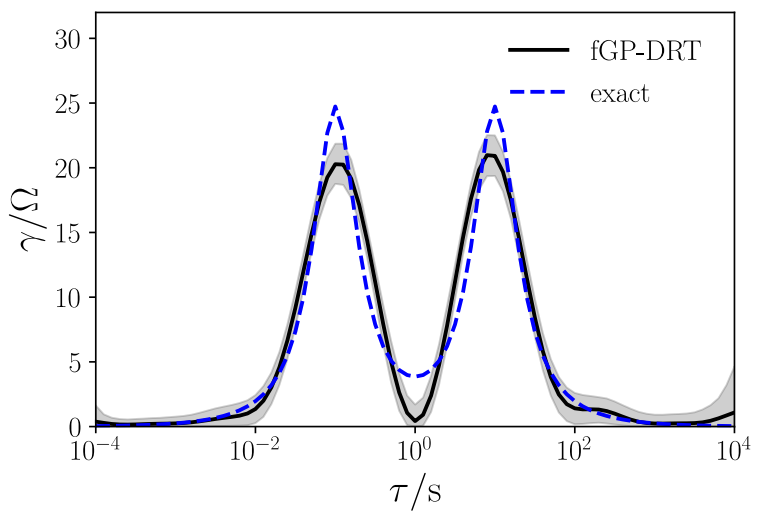

(e)
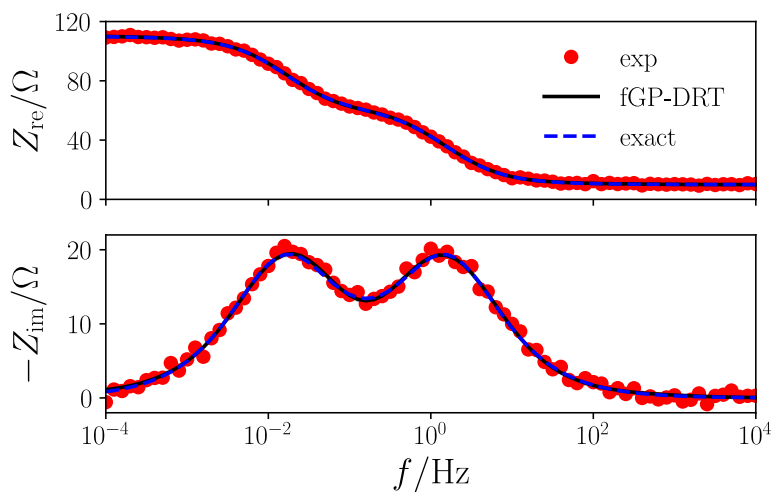

(b)

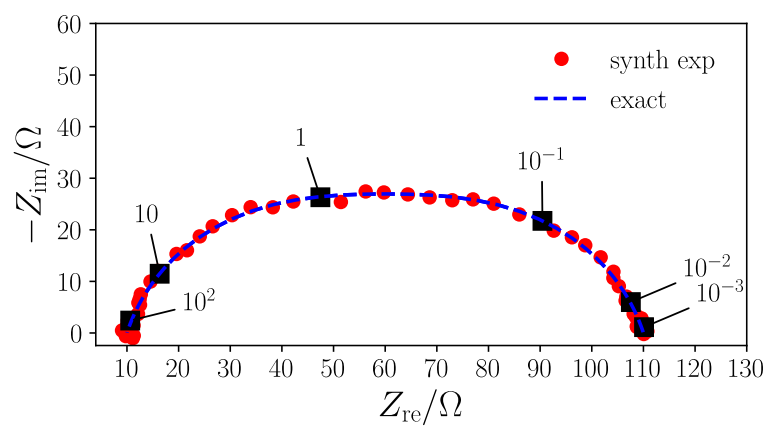

(d)

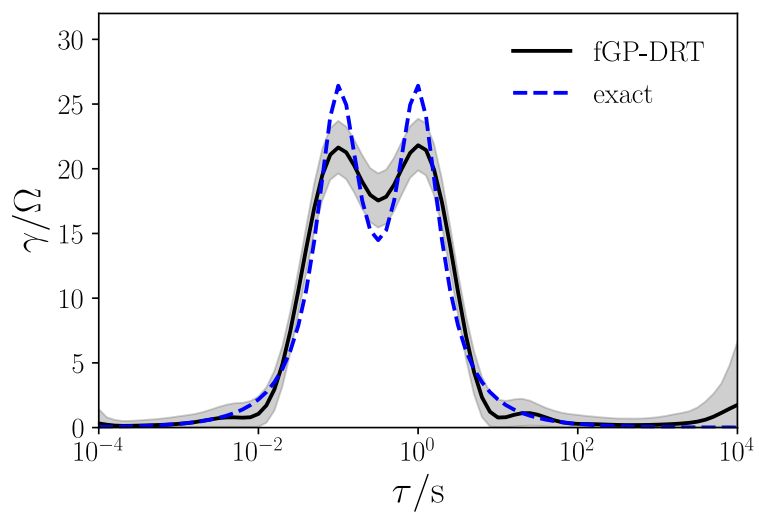

(f)
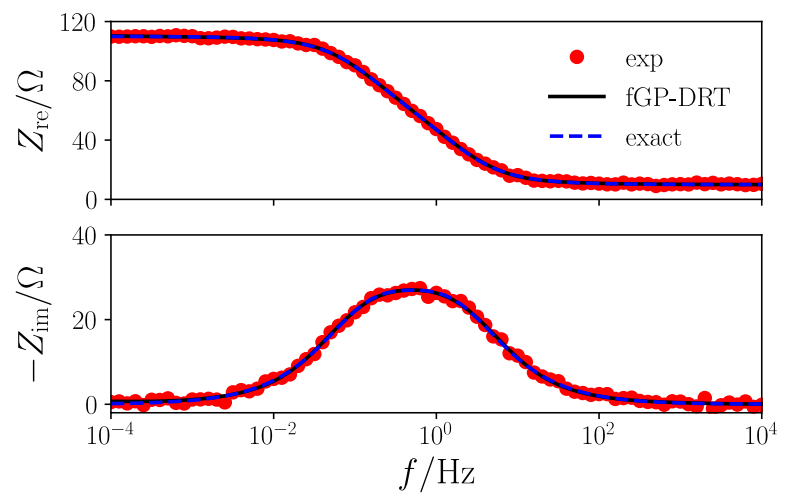

Figure 8: Nyquist plot of the $2 \times$ ZARC model with $\sigma_{n}^{\exp }=0.5 \Omega$ for (a) $\tau_{1}=0.1 \mathrm{~s}$ and $\tau_{2}=10 \mathrm{~s}$ and (b) $\tau_{1}=0.1 \mathrm{~s}$ and $\tau_{2}=1 \mathrm{~s}$. Corresponding DRT recovered using the fGP-DRT method under the non-negativity constraint are shown in panels (c) and (d), and real and imaginary parts of the impedance are reported in panels (e) and (f). 


\subsubsection{Piecewise Constant and Fractal Models}

We tested how the fGP-DRT model handles discontinuities in the DRT. To that end, the piecewise constant (PWC) $[36,63]$ and the fractal $[3,31,36,63]$ models were used. The exact impedance and the corresponding DRT of the PWC model are given by $[36,63]$

$$
\begin{gathered}
Z_{\text {exact }}(f)=R_{\infty}+\frac{R_{\mathrm{ct}}}{\log \left(\frac{\tau_{1}}{\tau_{0}}\right)}\left[\log \left(1-\frac{i}{2 \pi f \tau_{0}}\right)-\log \left(1-\frac{i}{2 \pi f \tau_{1}}\right)\right] \\
\gamma(\log \tau)=\frac{R_{\mathrm{ct}}}{\log \left(\frac{\tau_{1}}{\tau_{0}}\right)}\left(H\left(\tau-\tau_{0}\right)-H\left(\tau-\tau_{1}\right)\right)
\end{gathered}
$$

where $H(\tau)$ is the Heaviside function. For the fractal model $[3,31,36,63]$

$$
\begin{gathered}
Z_{\text {exact }}(f)=R_{\infty}+\frac{R_{\mathrm{ct}}}{\left(1+i 2 \pi f \tau_{0}\right)^{\phi}} \\
\gamma(\log \tau)=\left\{\begin{array}{c}
\frac{R_{\mathrm{ct}}}{\pi} \sin (\phi \pi)\left(\frac{\tau}{\tau_{0}-\tau}\right)^{\phi} \text { if } \tau<\tau_{0} \\
0 \quad \text { otherwise }
\end{array}\right.
\end{gathered}
$$

Panels (a), (c), and (e) of Figure 9 show the Nyquist plot, recovered DRT (under non-negativity constraint), and real and imaginary parts of the recovered impedance for the PWC model, respectively. Panels (b), (d), and (f) of Figure 9 depict the Nyquist plot, recovered DRT, and real and imaginary components of the impedance, respectively, for the fractal model. For both cases, the fGP-DRT model satisfactorily regresses the EIS data and is able to capture approximately the location of the discontinuities. 
(a)

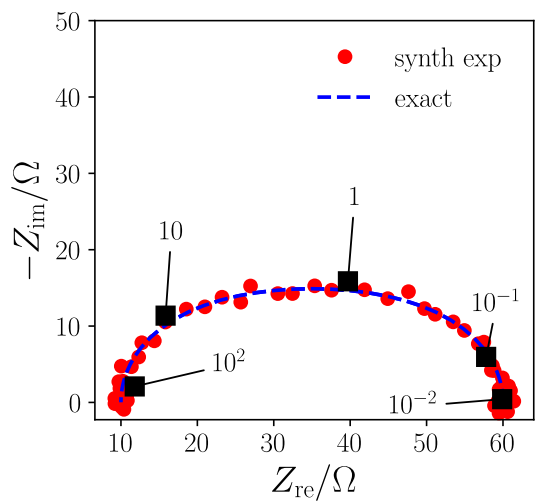

(c)

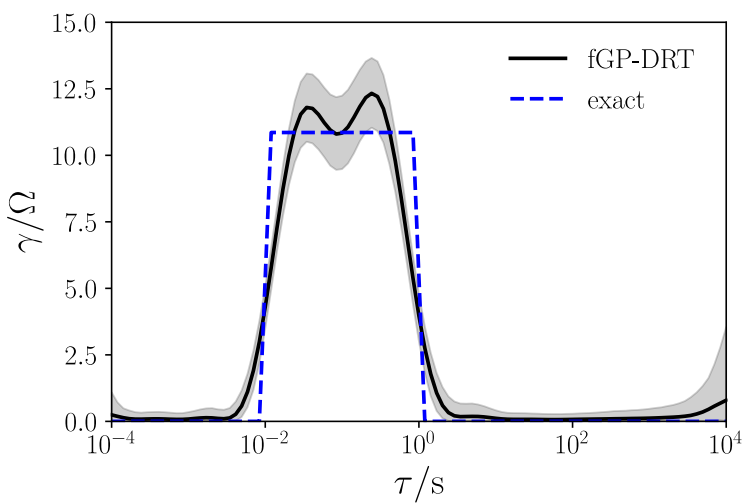

(e)
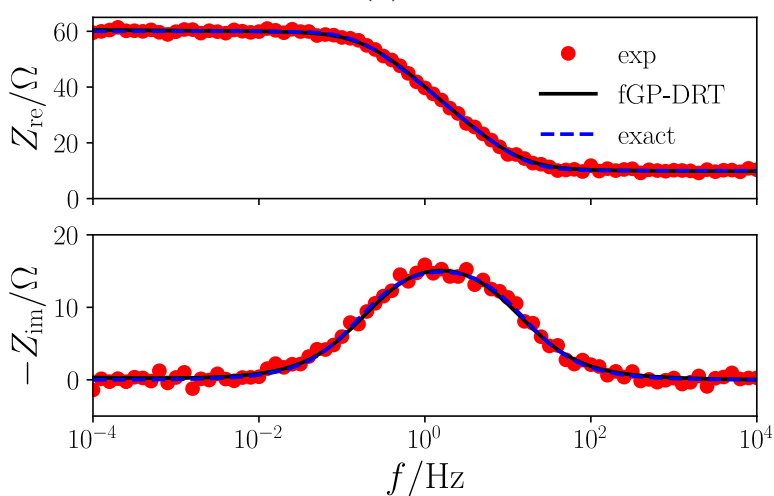

(b)

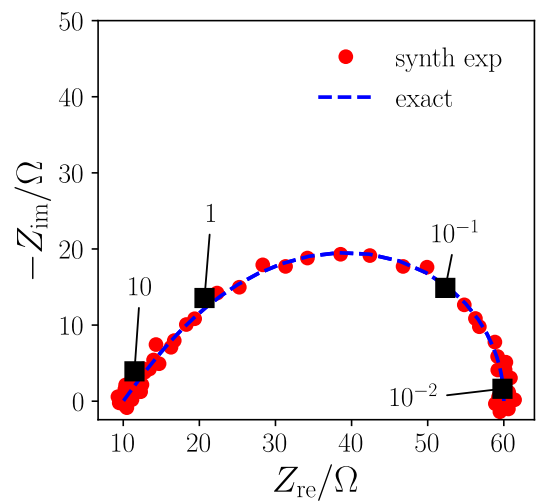

(d)

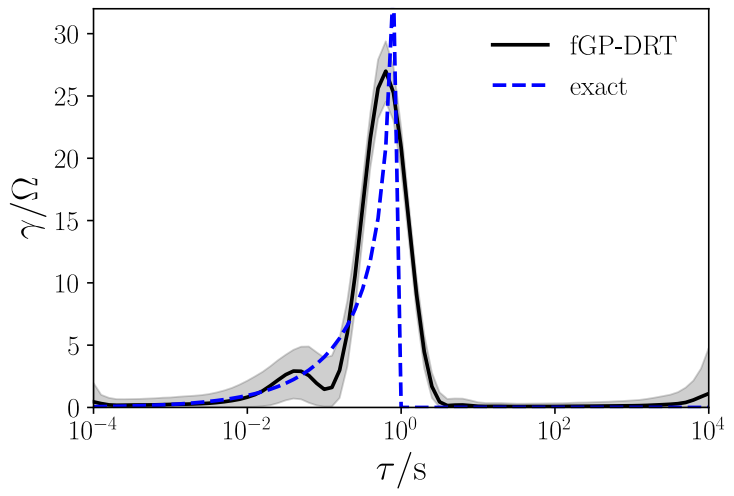

(f)
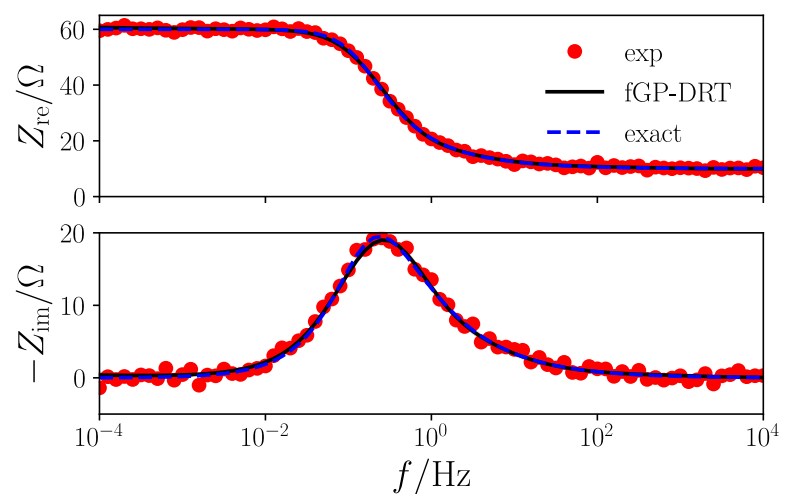

Figure 9: Nyquist plot of the (a) PWC model and (b) the fractal model $\left(\sigma_{n}^{\exp }=0.5 \Omega\right.$ ). Corresponding DRT recovered using the fGP-DRT method under the non-negativity constraint (c) and (d), and real and imaginary parts of the impedance (e) and (f). 


\subsection{Real Experiments}

Having tested the consistency of the fGP-DRT model with synthetic experiments, we evaluated its performance against real data from three symmetric solid oxide fuel cells and two batteries.

\subsubsection{Fuel Cells}

\subsubsection{Ba0.95La0.05FeO3- $-\delta$-based Symmetric Solid Oxide Cells}

EIS data was obtained from symmetric cells with $\mathrm{Ba}_{0.95} \mathrm{La}_{0.05} \mathrm{FeO}_{3-\delta}$ (BLF) [64] as the electrode material and samarium-doped ceria as the electrolyte. The tests were performed in the frequency range from $100 \mathrm{mHz}$ to $20 \mathrm{kHz}$ with five points per decade using a VSP potentiostat (BioLogic). The symmetric cell was tested at 500 and $550{ }^{\circ} \mathrm{C}$ in an atmosphere consisting of $\mathrm{N}_{2}$ and $\mathrm{O}_{2}$ with an oxygen partial pressure $\mathrm{pO}_{2}=60 \%$. The experimental data for each temperature was regressed against a $2 \times Z A R C$ (the best-fitting parameters are reported in Table S3). The regressed ECM and deconvolved fGP-DRT model match closely, as shown in Figure 10(a) and (b). Figure 10(c) and (d) present the DRT recovered using the fGP-DRT model and the ECM at 500 and $550{ }^{\circ} \mathrm{C}$, respectively. The two models appear to be consistent with small deviations in the DRT for $\tau>$ $100 \mathrm{~s}$.

Additionally, we considered the EIS of a symmetric cell with samarium-doped ceria as the electrolyte and the electrode consisting of a composite containing $\mathrm{BLF}$ and $\mathrm{Ag}_{2} \mathrm{O}$ (molar ratio 20:1) [65]. EIS data was collected at 550 and $700{ }^{\circ} \mathrm{C}$ in a mixture of $\mathrm{N}_{2}$ and $\mathrm{O}_{2}$ with $\mathrm{pO}_{2}=60 \%$. We found that the fGP-DRT is consistent with the ECM model (Figure S7), except for a small deviation for $\tau>10$ s (Figure S7(d)). 
(a)

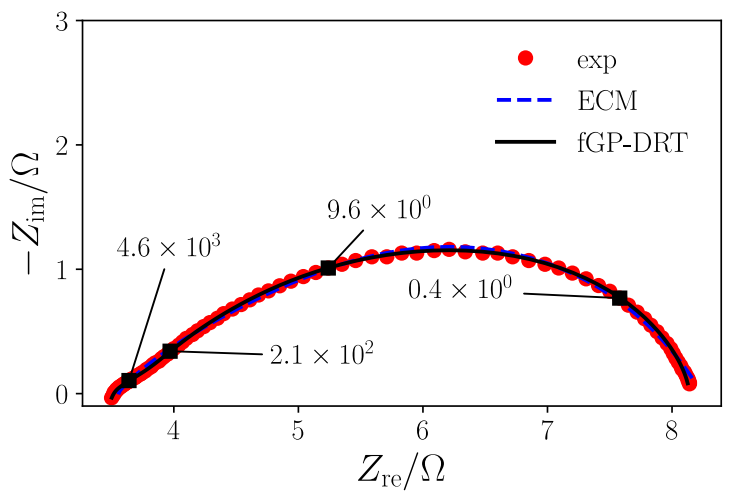

(c)

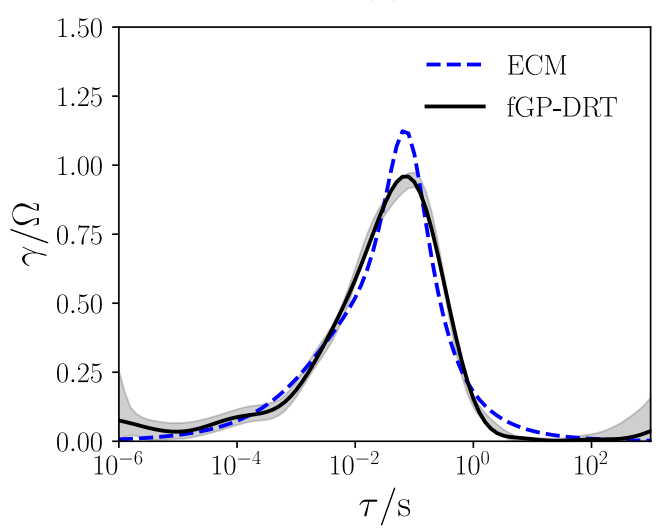

(e)
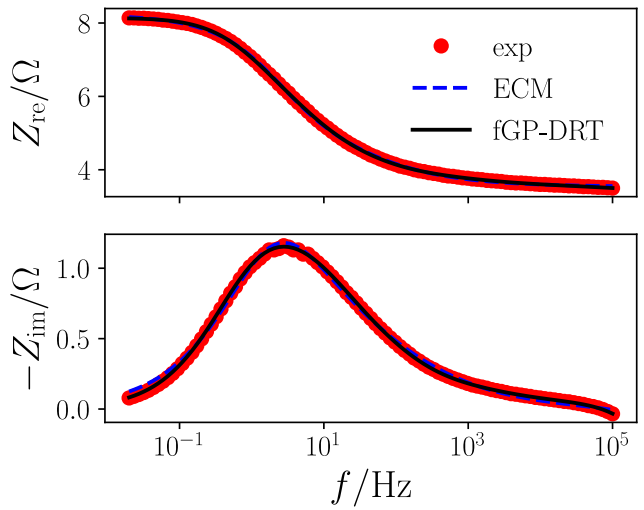

(b)

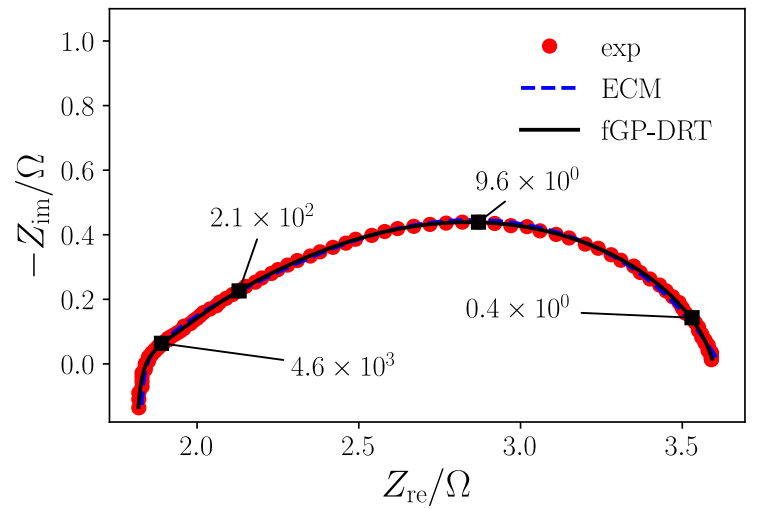

(d)

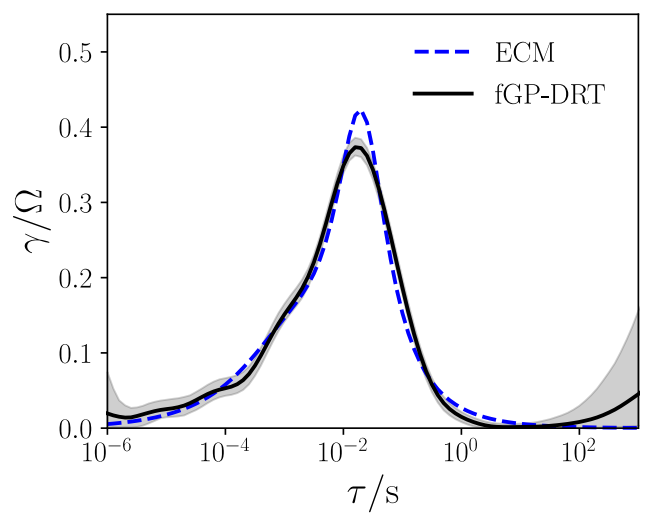

(f)
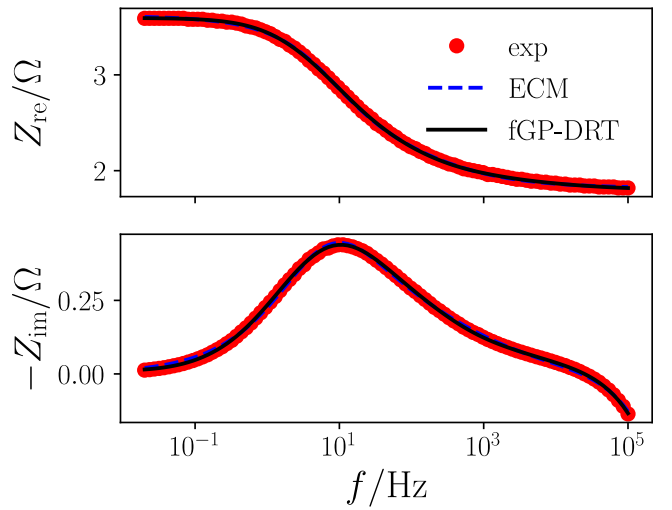

Figure 10: Nyquist plot of the BLF for $\mathrm{pO}_{2}=60 \%$ at (a) $500{ }^{\circ} \mathrm{C}$ and (b) $550{ }^{\circ} \mathrm{C}$ with the deconvolved fGP-DRT model and the regressed ECM. Corresponding (c) \& (d) DRT and (e) \& (f) real and imaginary parts of the impedance recovered using fGP-DRT method. Results of the regressed ECM are also shown for comparison. 


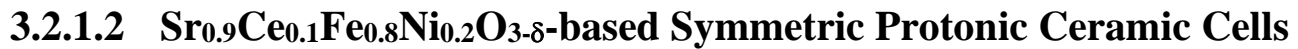

We analyzed the EIS data from a symmetric cell with $\mathrm{Sr}_{0.9} \mathrm{Ce}_{0.1} \mathrm{Fe}_{0.8} \mathrm{Ni}_{0.2} \mathrm{O}_{3-\delta}$ (SCFN) as the electrode material and $\mathrm{BaZr}_{0.1} \mathrm{Ce}_{0.7} \mathrm{Y}_{0.2-\mathrm{x}} \mathrm{O}_{3-\delta}$ as the electrolyte. The cell was tested at $500{ }^{\circ} \mathrm{C}$ in two atmospheres, namely 97\% Air-3\% $\mathrm{H}_{2} \mathrm{O}$ and 94\% Air-6\% $\mathrm{H}_{2} \mathrm{O}$ [67]. The data was obtained for frequencies ranging from $0.01 \mathrm{~Hz}$ to $200 \mathrm{kHz}$ and a $10 \mathrm{mV}$ amplitude of the input voltage. The measured EIS data was regressed against a $2 \times Z$ ZARC ECM whose parameters are reported in Table S4. The Nyquist plots of the SCFN with the deconvolved fGP-DRT model and the regressed ECM are presented in Figure 11(a) and (b), respectively. The two models match closely for both atmospheric conditions. 
(a)

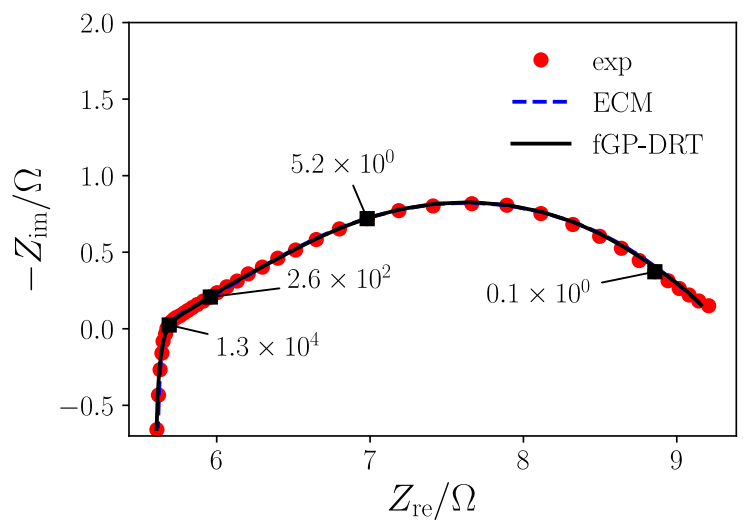

(c)

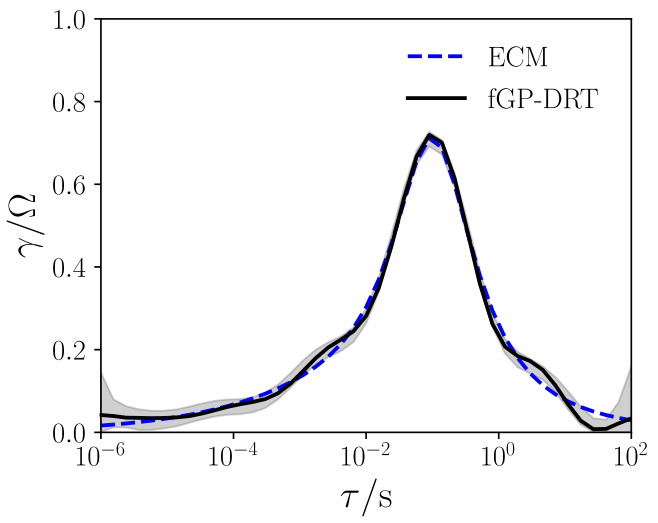

(e)
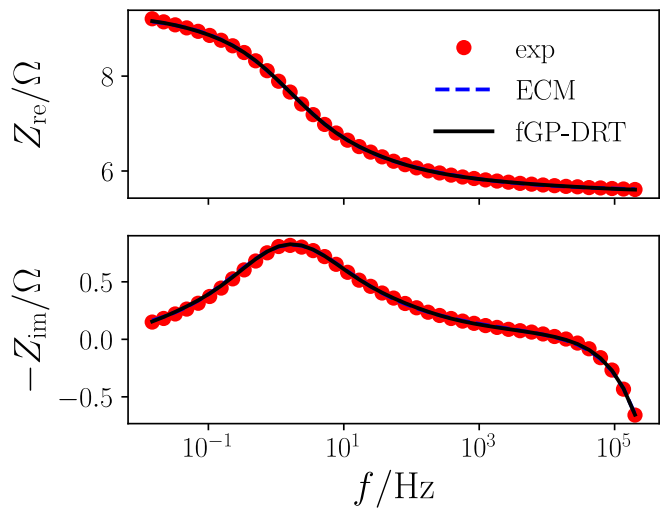

(b)

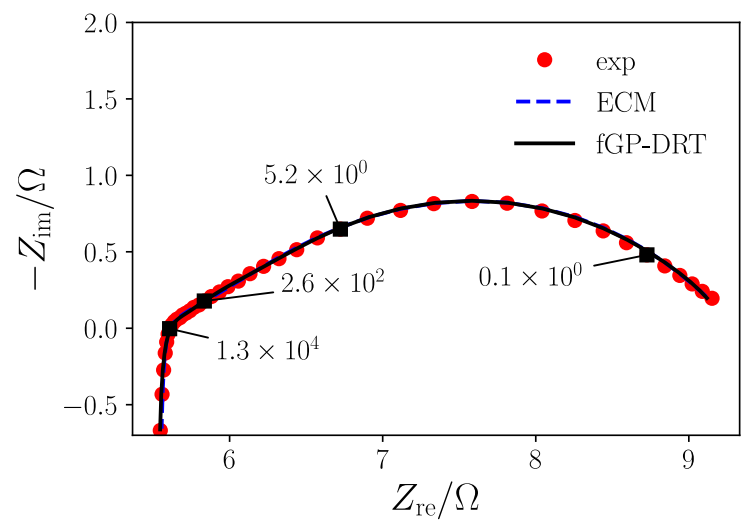

(d)

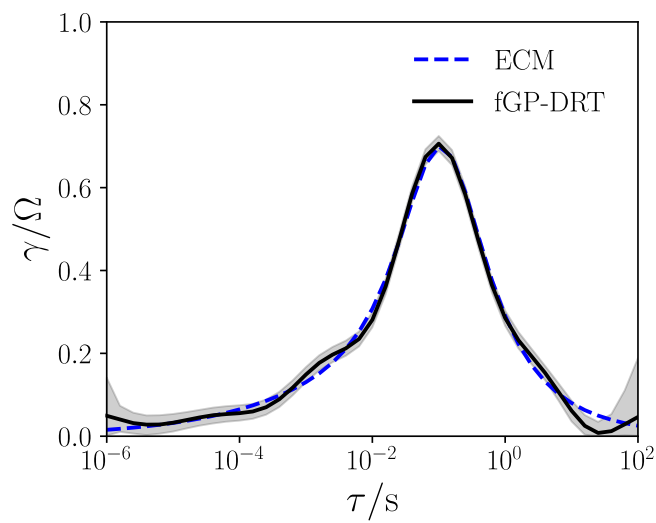

(f)
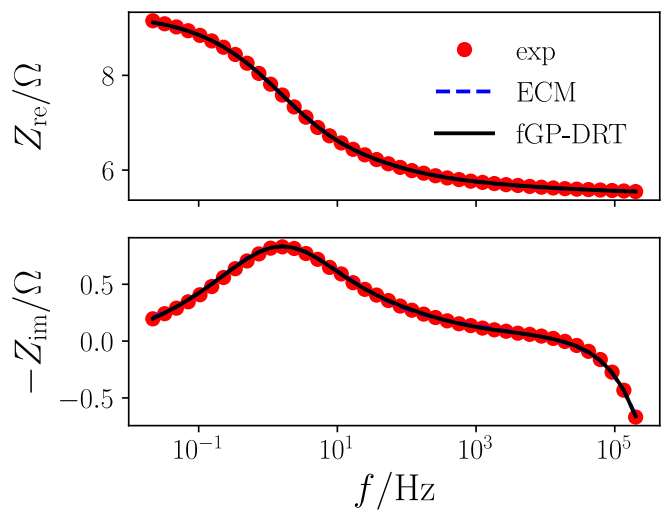

Figure 11: Nyquist plot of the SCFN-based cell at $500{ }^{\circ} \mathrm{C}$ at (a) $3 \% \mathrm{H}_{2} \mathrm{O}$ and (b) $6 \% \mathrm{H}_{2} \mathrm{O}$ with the deconvolved fGP-DRT model and the regressed ECM. Corresponding (c) and (d) DRT and real and imaginary parts of the impedance (e) and (f) recovered using the fGP-DRT method under nonnegativity constraint. Results using the regressed ECM are also shown for comparison. 


\subsubsection{Lithium-Ion Batteries}

\subsubsection{Composite Polymer Electrolytes}

We analyzed batteries with $\mathrm{Li}$-metal as the anode, a composite polymer electrolyte, and $\mathrm{LiFePO}_{4}$ as the cathode $[68,69]$. The EIS spectrum was regressed using the fGP-DRT model and a $3 \times$ ZARC ECM (the obtained ECM parameters are reported in Table S5). The Nyquist plot, recovered DRT with the regressed ECM, and real and imaginary parts of the impedance are displayed in the left panel of Figure S8. We observe that the DRT from the fGP-DRT and the ECM are consistent and that the fGP-DRT matches closely the experimental impedance.

\subsubsection{Solid-like Dual-salt Polymer Electrolytes}

We used experimental data obtained from a battery with the same anode and cathode as used in composite polymer electrolyte (see Section 3.2.2.1 above), and a solid-like dual-salt polymer electrolyte [67-70]. EIS experiments were carried out for frequencies between $1 \mathrm{~Hz}$ and $7 \mathrm{MHz}$. We repeated the same procedure as described Section 3.2.2.1, the results are displayed in the right panel of Figure S8. As above, the DRT obtained using the ECM and fGP-DRT method are consistent and the experimental impedance is closely regressed by the fGP-DRT method. 


\section{Conclusion}

In this work, we developed a novel fGP-DRT model based on a finite GP approximation that accurately deconvolves the DRT from EIS data. The new model inherits several traits of the previously developed GP-DRT since: 1) it assumes that the DRT is a GP; 2) analysis of synthetic and real experiments shows that this method is consistent; 3) its hyperparameters can be selected by maximizing the experimental evidence; and 4) the fGP-DRT is robust against experimental noise and data truncation. In addition, the fGP-DRT model outperforms the GP-DRT as it can: 1) be constrained to produce only non-negative DRTs; and 2) use both the real and imaginary parts of the impedance. In short, this article develops a new method for the probabilistic analysis of EIS spectra, opening up new research avenues that leverage finite GPs for DRT deconvolution.

\section{Code Availability}

Code for tutorial examples is available at https://github.com/ciuccislab/fGP-DRT

\section{Author Credit Statement}

Adeleke Maradesa: Extension and validation of software, Methodology, Experimental Analysis, Writing - Original Draft, Writing - Editing. Baptiste Py: Investigation, Writing - Review \&

Editing. Emanuel Quattrocchi: Review \& Editing. Francesco Ciucci: Software creation and development, Conceptualization, Methodology, Theory development, Investigation, Resources, Editing, Funding acquisition, Project administration

\section{Declaration of Competing Interest}

None 


\section{Acknowledgements}

The authors gratefully acknowledge the Research Grant Council of Hong Kong for support through the projects 16206019 and 16201820. A. Maradesa and E. Quattrocchi kindly thank the Hong Kong PhD. Fellowship Scheme for financial support. 


\section{List of Symbols}

\begin{tabular}{|c|l|}
\hline $\boldsymbol{\Gamma}$ & Covariance matrix \\
\hline $\boldsymbol{\theta}$ & Vector of random experimental errors \\
\hline $\boldsymbol{\mu}_{\mathrm{x}}$ & Vector of hyperparameters \\
\hline $\boldsymbol{\Sigma}_{\mathrm{x}}$ & Covariance matrix \\
\hline$\gamma(\log \tau)$ & Distribution of relaxation times \\
\hline$\Delta$ & Difference between any two collocation points \\
\hline$\xi$ & Logarithm of the relaxation time \\
\hline$\sigma_{f}$ & Variance of the squared-exponential kernel \\
\hline$\sigma_{L}$ & Standard deviation of the inductance \\
\hline$\sigma_{n}$ & Standard deviation of the random error \\
\hline$\sigma_{n}^{\exp }$ & Standard deviation of the experimental noise \\
\hline$\sigma_{R}$ & Standard deviation of the resistance \\
\hline$\tau$ & Relaxation time \\
\hline$\tau_{0}$ & Characteristic time of the constant phase element \\
\hline$\phi$ & Parameter of the constant phase element \\
\hline$\phi_{n}$ & \\
\hline & \\
\hline & \\
\hline & \\
\hline &
\end{tabular}

\begin{tabular}{|c|c|}
\hline $\boldsymbol{I}$ & $2 M \times 2 M$ identity matrix \\
\hline $\boldsymbol{K}$ & GP covariance matrix \\
\hline $\boldsymbol{x}$ & DRT vector \\
\hline $\boldsymbol{Z}$ & Impedance vector \\
\hline
\end{tabular}




\begin{tabular}{|c|c|}
\hline$Z_{\text {exp }}$ & Vector of the experimental impedance \\
\hline$f$ & Frequency \\
\hline$g(\tau)$ & Distribution function \\
\hline$H(\tau)$ & Heaviside function \\
\hline$k\left(\xi, \xi^{\prime}\right)$ & Kernel function \\
\hline$\ell$ & Length scale of the squared-exponential kernel \\
\hline$L(\boldsymbol{\theta})$ & Negative marginal log-likelihood \\
\hline$L_{0}$ & Inductance \\
\hline$m(\xi)$ & GP mean function \\
\hline$M$ & Number of frequencies \\
\hline$N$ & Number of collocation points \\
\hline$r^{2}$ & Normalized squared residuals \\
\hline$R_{\mathrm{ct}}$ & Charge transfer resistance \\
\hline$R_{\infty}$ & Ohmic resistance \\
\hline
\end{tabular}

\section{List of Abbreviations}

\begin{tabular}{|c|c|}
\hline BLF & $\mathrm{Ba}_{0.95} \mathrm{La}_{0.05} \mathrm{FeO}_{3-\delta}$ \\
\hline DRT & Distribution of relaxation times \\
\hline ECM & Equivalent circuit model \\
\hline EIS & Flectrochemical impedance spectroscopy \\
\hline fGP-DRT & Gaussian process \\
\hline GP & Gaussian process distribution of relaxation times \\
\hline GP-DRT & Piecewise constant \\
\hline PWC & $\mathrm{Sr}_{0.9} \mathrm{Ce}_{0.1} \mathrm{Fe}_{0.8} \mathrm{Ni}_{0.2} \mathrm{O}_{3-\delta}$ \\
\hline SCFN & \\
\hline
\end{tabular}




\section{References}

[1] J. Liu, F. Ciucci, The Gaussian process distribution of relaxation times: A machine learning tool for the analysis and prediction of electrochemical impedance spectroscopy data, Electrochim. Acta. 331 (2020) 135-316.

[2] M.A. Danzer, Generalized distribution of relaxation times analysis for the characterization of impedance spectra, Batteries. 5 (2019) 5-53.

[3] B.A. Boukamp, Derivation of a distribution function of relaxation times for the (fractal) finite length warburg., Electrochim. Acta. 252 (2017) 154-163.

[4] F. Dion, A. Lasia, The use of regularization methods in the deconvolution of underlying distributions in electrochemical processes, J. Electroanal. Chem. 475 (1999) 28-37.

[5] Y. Zheng, Z. Shi, D. Guo, H. Dai, X. Han, A simplification of the time-domain equivalent circuit model for lithium-ion batteries based on low-frequency electrochemical impedance spectra, J. Power Sources. 489 (2021) $229-505$.

[6] B.A. Boukamp, A. Rolle, Use of a distribution function of relaxation times (DFRT) in impedance analysis of SOFC electrodes, Solid State Ion. 314 (2018) 103-111.

[7] B.A. Boukamp, A. Rolle, R.N. Vannier, R.K. Sharma, E. Djurado, Electrostatic spray deposited $\mathrm{Ca}_{3} \mathrm{Co}_{4} \mathrm{O}_{9+\delta}$ and $\mathrm{Ca}_{3} \mathrm{Co}_{4} \mathrm{O}_{9+\delta} / \mathrm{Ce}_{0.95} \mathrm{Gd}_{0.1} \mathrm{O}_{1.95}$ cathodes for SOFC, Electrochim. Acta. 362 (2020) 137-142.

[8] B.A. Boukamp, Distribution (function) of relaxation times, successor to complex nonlinear least squares analysis of electrochemical impedance spectroscopy?, J. Phys. Energy. 2 (2020) 104-201.

[9] B.-Y. Chang, S.-M. Park, Electrochemical impedance spectroscopy, Annu. Rev. Anal. Chem. 3 (2010) 207-229. 
[10] J. Fleig, Impedance Spectroscopy on Solids: The limits of serial equivalent circuit models, J. Electroceramics. 13 (2004) 637-644.

[11] A. Leonide, V. Sonn, A. Weber, E. Ivers-Tiffée, Evaluation and Modeling of the Cell Resistance in Anode-Supported Solid Oxide Fuel Cells, J. Electrochem. Soc. 155 (2008) B36-B41.

[12] M.E. Orazem, B. Tribollet, Electrochemical impedance spectroscopy, Wiley, Hoboken, N.J, 2008.

[13] H.D. Yoo, J.H. Jang, J.H. Ryu, Y. Park, S.M. Oh, Impedance analysis of porous carbon electrodes to predict rate capability of electric double-layer capacitors, J. Power Sources. 267 (2014) 411-420.

[14] K.C. Kam, A. Mehta, J.T. Heron, M.M. Doeff, Electrochemical and physical properties of Ti-Substituted layered nickel manganese cobalt oxide (NMC) Cathode Materials, J. Electrochem. Soc. 159 (2012) A1383-A1392.

[15] E. Quattrocchi, T.H. Wan, A. Curcio, S. Pepe, M.B. Effat, F. Ciucci, A general model for the impedance of batteries and supercapacitors: The non-linear distribution of diffusion times, Electrochim. Acta. 324 (2019) 134-853.

[16] S. Buller, M. Thele, R.W.A.A. DeDoncker, E. Karden, Impedance-based simulation models of supercapacitors and Li-ion batteries for power electronic applications, IEEE Trans. Ind. Appl. 41 (2005) 742-747.

[17] E. IVERS-TIFF\&Eacute;E, A. Weber, Evaluation of electrochemical impedance spectra by the distribution of relaxation times, J. Ceram. Soc. Jpn. 125 (2017) 193-201.

[18] C.H. Chen, J. Liu, K. Amine, Symmetric cell approach and impedance spectroscopy of high power lithium-ion batteries, J. Power Sources. 96 (2001) 321-328. 
[19] W. Waag, S. Käbitz, D.U. Sauer, Experimental investigation of the lithium-ion battery impedance characteristic at various conditions and aging states and its influence on the application, Appl. Energy. 102 (2013) 885-897.

[20] Z. He, F. Mansfeld, Exploring the use of electrochemical impedance spectroscopy (EIS) in microbial fuel cell studies, Energy Env. Sci. 2 (2009) 215-219.

[21] M.J. Jørgensen, M. Mogensen, Impedance of solid oxide fuel cell LSM/YSZ composite cathodes, J. Electrochem. Soc. 148 (2001) A433-A442.

[22] A. Weiß, S. Schindler, S. Galbiati, M.A. Danzer, R. Zeis, Distribution of relaxation times analysis of high-temperature PEM fuel cell impedance spectra, Electrochim. Acta. 230 (2017) 391-398.

[23] H. Sumi, H. Shimada, Y. Yamaguchi, T. Yamaguchi, Y. Fujishiro, Degradation evaluation by distribution of relaxation times analysis for microtubular solid oxide fuel cells, Electrochim. Acta. 339 (2020) 135-913.

[24] F. Ciucci, Modeling electrochemical impedance spectroscopy, Curr. Opin. Electrochem. 13 (2019) 132-139.

[25] J.G. Lyagaeva, G.K. Vdovin, D.A. Medvedev, Distinguishing bulk and grain boundary transport of a proton-conducting electrolyte by combining equivalent circuit scheme and distribution of relaxation times analyses, J. Phys. Chem. C. 123 (2019) 21993-21997.

[26] D. Clematis, S. Presto, M.P. Carpanese, A. Barbucci, F. Deganello, L.F. Liotta, C. Aliotta, M. Viviani, Distribution of relaxation times and equivalent circuits analysis of $\mathrm{Ba}_{0.5} \mathrm{Sr}_{0.5} \mathrm{Co}_{0.8} \mathrm{Fe}_{0.2} \mathrm{O}_{3-\delta}$, Catalysts. 9 (2019) 441. 
[27] S.C. Page, A.H. Anbuky, S.P. Krumdieck, J. Brouwer, Test method and equivalent circuit modeling of a PEM fuel cell in a passive state, IEEE Trans. Energy Convers. 22 (2007) 764773.

[28] M.B. Effat, F. Ciucci, Bayesian and hierarchical bayesian based regularization for deconvolving the distribution of relaxation times from electrochemical impedance spectroscopy data, Electrochim. Acta. 247 (2017) 1117-1129.

[29] D. Kim, E.S. Muckley, N. Creange, T.H. Wan, M.H. Ann, E. Quattrocchi, R.K. Vasudevan, J.H. Kim, F. Ciucci, I.N. Ivanov, S.V. Kalinin, M. Ahmadi, Exploring transport behavior in hybrid perovskites solar cells via machine learning analysis of environmental-dependent impedance spectroscopy, Adv. Sci. 8 (2021) 200-2510.

[30] D.A. Harrington, P. van den Driessche, Mechanism and equivalent circuits in electrochemical impedance spectroscopy, Electrochim. Acta. 56 (2011) 8005-8013.

[31] X. Li, M. Ahmadi, L. Collins, S.V. Kalinin, Deconvolving distribution of relaxation times, resistances and inductance from electrochemical impedance spectroscopy via statistical model selection: Exploiting structural-sparsity regularization and data-driven parameter tuning, Electrochim. Acta. 313 (2019) 570-583.

[32] D. Klotz, J.P. Schmidt, A. Kromp, A. Weber, E. Ivers-Tiffée, The Distribution of relaxation times as beneficial tool for equivalent circuit modeling of fuel cells and batteries, ECS Trans. 41 (2019) 25-33.

[33] E. Tuncer, M. Wegener, R. Gerhard-Multhaupt, Distribution of relaxation times in $\alpha$-phase polyvinylidene fluoride, J. Non-Cryst. Solids. 351 (2005) 2917-2921.

[34] A. Mertens, J. Granwehr, Two-dimensional impedance data analysis by the distribution of relaxation times, J. Energy Storage. 13 (2017) 401-408. 
[35] J. Xia, C. Wang, X. Wang, L. Bi, Y. Zhang, A perspective on DRT applications for the analysis of solid oxide cell electrodes, Electrochim. Acta. 349 (2020) 136328.

[36] F. Ciucci, C. Chen, Analysis of electrochemical impedance spectroscopy data using the distribution of relaxation times: a bayesian and hierarchical bayesian approach, Electrochim. Acta. 167 (2015) 439-454.

[37] M. Eckert, L. Kolsch, S. Hohmann, Fractional algebraic identification of the distribution of relaxation times of battery cells, in: 2015 54th IEEE Conf. Decis. Control CDC, IEEE, Osaka, 2015: pp. 2101-2108.

[38] S. Effendy, J. Song, M.Z. Bazant, Analysis, design, and generalization of electrochemical impedance spectroscopy (EIS) inversion algorithms, J. Electrochem. Soc. 167 (2020) 106508.

[39] J. Kaipio, E. Somersalo, Statistical inverse problems: discretization, model reduction and inverse crimes, J. Comput. Appl. Math. 198 (2007) 493-504.

[40] D. Calvetti, E. Somersalo, Inverse problems: from regularization to bayesian inference, WIREs Comput. Stat. 10 (2018).

[41] M. Saccoccio, T.H. Wan, C. Chen, F. Ciucci, Optimal regularization in distribution of relaxation times applied to electrochemical impedance spectroscopy: ridge and lasso regression methods - a theoretical and experimental study, Electrochim. Acta. 147 (2014) $470-482$.

[42] A.N. Tikhonov, A.V. Goncharsky, V.V. Stepanov, A.G. Yagola, Numerical methods for the solution of ill-Posed problems, Springer Netherlands, Dordrecht, 1995. 
[43] T. Paul, P.W. Chi, P.M. Wu, M.K. Wu, Computation of distribution of relaxation times by Tikhonov regularization for Li ion batteries: usage of L-curve method, Sci. Rep. 11 (2021) 12624.

[44] A.L. Gavrilyuk, D.A. Osinkin, D.I. Bronin, On a variation of the Tikhonov regularization method for calculating the distribution function of relaxation times in impedance spectroscopy, Electrochim. Acta. 354 (2020) 136-683.

[45] S. Hershkovitz, S. Tomer, S. Baltianski, Y. Tsur, ISGP: Impedance spectroscopy analysis using evolutionary programming procedure, ECS Trans. 33 (2019) 67-73.

[46] A.B. Tesler, D.R. Lewin, S. Baltianski, Y. Tsur, Analyzing results of impedance spectroscopy using novel evolutionary programming techniques, J. Electroceramics. 24 (2010) 245-260.

[47] E. Quattrocchi, T.H. Wan, A. Belotti, D. Kim, S. Pepe, S.V. Kalinin, M. Ahmadi, F. Ciucci, The deep-DRT: A deep neural network approach to deconvolve the distribution of relaxation times from multidimensional electrochemical impedance spectroscopy data, Electrochim. Acta. 392 (2021) 139010.

[48] P.C. Hansen, D.P. O'Leary, The Use of the L-Curve in the regularization of discrete ill-posed problems, SIAM J. Sci. Comput. 14 (1993) 1487-1503.

[49] T.H. Wan, M. Saccoccio, C. Chen, F. Ciucci, Influence of the discretization methods on the distribution of relaxation times deconvolution: implementing radial basis functions with DRTtools, Electrochim. Acta. 184 (2015) 483-499.

[50] Y. Zhang, Y. Chen, M. Yan, F. Chen, Reconstruction of relaxation time distribution from linear electrochemical impedance spectroscopy, J. Power Sources. 283 (2015) 464-477. 
[51] F. Ciucci, T. Carraro, W.C. Chueh, W. Lai, Reducing error and measurement time in impedance spectroscopy using model based optimal experimental design, Electrochim. Acta. 56 (2011) 5416-5434.

[52] A.K. Baral, Y. Tsur, Impedance spectroscopy of Gd-doped ceria analyzed by genetic programming (ISGP) method, Solid State Ion. 304 (2017) 145-149.

[53] T. Hörlin, Deconvolution and maximum entropy in impedance spectroscopy of noninductive systems, Solid State Ion. 107 (1998) 241-253.

[54] F. Ciucci, The Gaussian process hilbert transform (GP-HT): testing the consistency of electrochemical impedance spectroscopy data, J. Electrochem. Soc. 167 (2020) 126503.

[55] A.F. López-Lopera, Bachoc, Finite-dimensional Gaussian approximation with linear inequality constraints, SIAMASA J. Uncertain. Quantif. 6 (2018) 1224-1255.

[56] C.E. Rasmussen, C.K.I. Williams, Gaussian processes for machine learning, MIT Press, Cambridge, Mass, 2006.

[57] K.P. Murphy, Machine learning: a probabilistic perspective, MIT Press, Cambridge, MA, 2012.

[58] A. Pakman, L. Paninski, Exact Hamiltonian Monte Carlo for Truncated Multivariate Gaussians, J. Comput. Graph. Stat. 23 (2014) 518-542.

[59] A.B. Abdessalem, N. Dervilis, D.J. Wagg, K. Worden, Automatic kernel selection for Gaussian processes Regression with approximate bayesian computation and sequential monte carlo, Front. Built Environ. 3 (2017) 52.

[60] C.E. Rasmussen, C.K.I. Williams, Gaussian processes for machine learning, MIT Press, Cambridge, Mass, 2006. 
[61] D. Clematis, T. Ferrari, A. Bertei, A.M. Asensio, M.P. Carpanese, C. Nicolella, A. Barbucci, On the stabilization and extension of the distribution of relaxation times analysis, Electrochim. Acta. 391 (2021) 138916.

[62] F. Ciucci, Revisiting parameter identification in electrochemical impedance spectroscopy: Weighted least squares and optimal experimental design, Electrochim. Acta. 87 (2013) 532545.

[63] E. Barsoukov, J.R. Macdonald, eds., Impedance spectroscopy: theory, experiment, and applications, Third edition, Wiley, Hoboken, NJ, 2018.

[64] J. Liu, J. Wang, A. Belotti, F. Ciucci, P-Substituted $\mathrm{Ba}_{0.95} \mathrm{La}_{0.05} \mathrm{FeO}_{3-\delta}$ as a Cathode Material for SOFCs, ACS Appl. Energy Mater. 2 (2019) 5472-5480.

[65] A. Belotti, J. Liu, A. Curcio, J. Wang, Z. Wang, E. Quattrocchi, M.B. Effat, F. Ciucci, Introducing $\mathrm{Ag}$ in $\mathrm{Ba}_{0.9} \mathrm{La}_{0.1} \mathrm{FeO}_{3-\delta}$ : Combining cationic substitution with metal particle decoration, Mater. Rep. Energy. 1 (2021) 100018.

[66] R.P. O’Hayre, S.-W. Cha, W.G. Colella, F.B. Prinz, Fuel cell fundamentals, Third edition, John Wiley \& Sons Inc, Hoboken, New Jersey, 2016.

[67] Y. Song, J. Liu, Y. Wang, D. Guan, A. Seong, M. Liang, M.J. Robson, X. Xiong, Z. Zhang, G. Kim, Z. Shao, F. Ciucci, Nanocomposites: a new opportunity for developing highly active and durable bifunctional air electrodes for reversible protonic ceramic cells, Adv. Energy Mater. 11 (2021) 2101899.

[68] J. Yu, J. Liu, X. Lin, H.M. Law, G. Zhou, S.C.T. Kwok, M.J. Robson, J. Wu, F. Ciucci, A solid-like dual-salt polymer electrolyte for Li-metal batteries capable of stable operation over an extended temperature range, Energy Storage Mater. 37 (2021) 609-618. 
[69] Z. Dai, J. Yu, J. Liu, R. Liu, Q. Sun, D. Chen, F. Ciucci, Highly conductive and nonflammable composite polymer electrolytes for rechargeable quasi-solid-state Li-metal batteries, J. Power Sources. 464 (2020) 228182.

[70] S. Jiao, X. Ren, R. Cao, M.H. Engelhard, Y. Liu, D. Hu, D. Mei, J. Zheng, W. Zhao, Q. Li, N. Liu, B.D. Adams, C. Ma, J. Liu, J.-G. Zhang, W. Xu, Stable cycling of high-voltage lithium metal batteries in ether electrolytes, Nat. Energy. 3 (2018) 739-746. 